\title{
DIGITALCOMMONS
}

$@$ WAYNESTATE-

Wayne State University

5-1-2009

\section{Metric Regularity and Lipschitzian Stability of Parametric Variational Systems}

Francisco J. Aragón Artacho

University of Alicante, Spain, francisco.aragon@ua.es

Boris S. Mordukhovich

Wayne State University, boris@math.wayne.edu

\section{Recommended Citation}

Aragón Artacho, Francisco J. and Mordukhovich, Boris S., "Metric Regularity and Lipschitzian Stability of Parametric Variational Systems" (2009). Mathematics Research Reports. Paper 66.

http://digitalcommons.wayne.edu/math_reports/66 


\section{METRIC REGULARITY AND LIPSCHITZIAN STABILITY OF PARAMETRIC VARIATIONAL SYSTEMS}

FRANCISCO J. ARAGÓN ARTACHO and BORIS S. MORDUKHOVICH

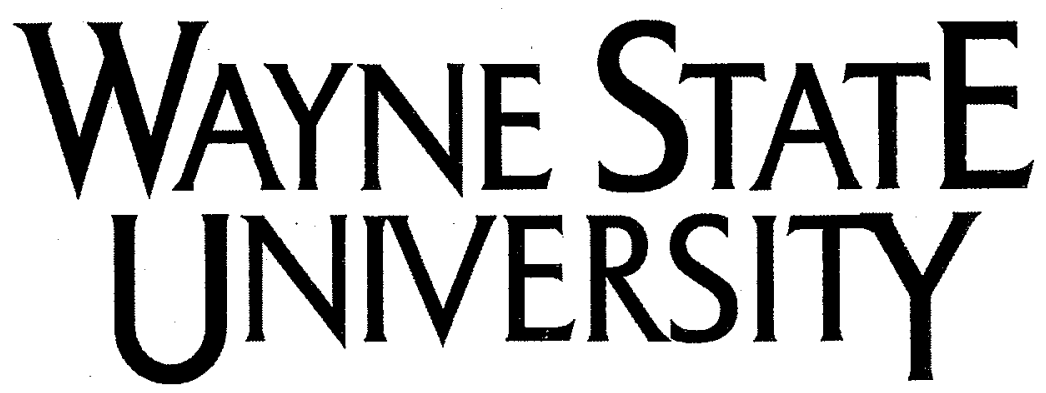

Detroit, MI 48202

Department of Mathematics

Research Report

2009 Series

\#5

This research was partly supported by the USA National Science Foundation 


\title{
METRIC REGULARITY AND LIPSCHITZIAN STABILITY OF PARAMETRIC VARIATIONAL SYSTEMS
}

\author{
FRANCISCO J. ARAGÓN ARTACHO ${ }^{1}$ and BORIS S. MORDUKHOVICH ${ }^{2}$
}

\begin{abstract}
The paper concerns the study of variational systems described by parameterized generalized equations/variational conditions important for many aspects of nonlinear analysis, optimization, and their applications. Focusing on the fundamental properties of metric regularity and Lipschitzian stability, we establish various qualitative and quantitative relationships between these properties for multivalued parts/fields of parametric generalized equations and the corresponding solution maps for them in the framework of arbitrary Banach spaces of decision and parameter variables.

Keywords: Variational analysis and optimization; Parametric variational systems; Generalized equations and variational inequalities; Metric regularity and subregularity; Lipschitzian stability; Calmness
\end{abstract}

\section{Introduction}

The main objective of this paper is to study some well-posedness properties for a large class of variational systems governed by parametric generalized equation in the sense of Robinson [20]:

$$
0 \in f(x, y)+Q(y)
$$

depending on the decision variable $y \in Y$ and the parameter variable $x \in X$ with a single-valued base mapping $f: X \times Y \rightarrow Z$ and a set-valued field mapping $Q: Y \Rightarrow Z$ between arbitrary Banach spaces. Formalism (1.1), known also as "variational condition" [23], has been well recognized as a convenient model for the study of many qualitative and quantitative aspects of variational analysis, optimization, equilibria, and their numerous applications; see, e.g., books $[5,12,17,23]$ and the references therein. Recall that model (1.1) encompasses, in particular, parameterized variational inequalities corresponding to the normal cone mapping $Q(y)=N(y ; \Omega)$ with a convex set $\Omega$, various complementarity problems, KKT (Karush-Kuhn-Tucker) systems of first-order optimality conditions in mathematical programming, etc.

Associating with (1.1) the parameter-dependent solution map $S: X \rightrightarrows Y$ given by

$$
S(x):=\{y \in Y \mid 0 \in f(x, y)+Q(y)\}
$$

we intend to establish relationships between certain fundamental well-posedness properties of the solution map (1.2) and those of the field mapping $Q$ of the generalized equation (1.1). Namely, we concentrate on two basic versions of metric regularity and Lipschitzian stability for $S$ for $Q$, which all play a crucial role in many areas of nonlinear analysis and its applications, particularly in their variational aspects; see the subsequent discussion in Section 2 . Our main results show that the metric regularity properties of $S$ at and around the points in question are equivalent, under appropriate surjectivity assumptions on the partial derivative of the base mapping $f$ with respect

\footnotetext{
${ }^{1}$ Department of Statistics and Operations Research, University of Alicante, 03071 Alicante, Spain (email: francisco.aragon@ua.es). Research of this author was partially supported by MICINN of Spain, grant MTM2008-06695C03-01 and program "Juan de la Cierva."

${ }^{2}$ Department of Mathematics, Wayne State University, Detroit, MI 48202, USA (email: boris@math.wayne.edu). Research of this author was partially supported by the USA National Science Foundation under grant DMS-0603846.
} 
to $x$ or the like, to the corresponding Lipschitzian properties of the field mapping $Q$, and vice versa. Note that these two lines of equivalence are independent of each other, since there is no symmetry between the mappings $S$ and $Q$ and/or their inverses. Besides these qualitative equivalence results, we derive quantitative relationships between the characteristic constants (exact bounds of moduli) associated with the metric regularity and Lipschitzian properties of $S$ and $Q$ involving also the corresponding data of the base mapping $f$ in (1.1).

In fact, the initial motivation for our study comes from the recent results in [18] discovering the failure of metric regularity around the points in question for solution maps (1.2) to some major classes of parametric generalized equations (1.1) due to the equivalence between this property and the Lipschitz-like/Aubin property of the corresponding field mappings $Q$ established in [6] on the base of coderivative analysis in Asplund spaces. This class of spaces can be described as Banach spaces, where all separable subspaces have separable duals. In this paper we extend, in particular, the aforementioned results of $[6,18]$ to the case of arbitrary Banach spaces employing a direct approach based on enhanced iterative processes of the Lyusternik-Graves type that does not use the coderivative characterizations of metric regularity and Lipschitzian stability and allows us in addition to derive tight relationships between the exact bounds of the corresponding moduli. The latter seems to be new even in finite dimensions. As already mentioned, the approach of this paper leads also to establishing new qualitative and quantitative relationships between the Lipschitz-like property of $S$ and the metric regularity of $Q$ around the corresponding points.

Furthermore, we explore the validity as well as the violation of similar relationships between "unstable" counterparts of the above metric regularity and Lipschitz-like properties of $S$ and $Q$ defined at (versus around) the points in questions and known also as "metric subregularity" and "calmness," respectively; see the exact definitions and more discussions in the next section.

The rest of the paper is organized as follows. Section 2 contains definitions of the basic properties under consideration, some preliminary results, and discussions.

Section 3 is devoted to establishing various relationships (mainly equivalences) between, on one hand, metric regularity of solutions maps $S$ to parametric generalized equations around and at the given points and, on the other hand, the corresponding Lipschitzian/calmness properties of field mappings $Q$ in (1.1). Quantitative results involving the exact bounds of the corresponding moduli in these properties together with appropriate characteristics of base mappings $f$ in (1.1) are derived simultaneously via the underlying iterative processes.

In Section 4 we apply the equivalence results of the previous section to make a conclusion on violating the metric regularity property and also its weak counterpart around the points in question for important classes of variational systems described via solution maps to parametric generalized equations with monotone as well as composite subdifferential fields in the general Banach space framework. We discuss the essence of this ill-posedness phenomenon and show that the results of this type do not hold for the at point counterpart of metric regularity, i.e., for metric subregularity.

The final Section 5 of the paper concerns establishing qualitative and quantitative relationships between, on one hand, the Lipschitzian/calmness properties of solution maps to parametric generalized equations and, on the other hand, the metric regularity/subregularity properties of field mappings in (1.1), i.e., we consider the reverse setting to Section 3. Besides deriving "positive" equivalence results in this direction independent of those in Section 3, it is shown here that there is no parallelism between these two settings in general. In particular, it is confirmed by examples that the equivalence between the at-point properties considered in this section holds to much lesser extent in comparison with the results established in Section 3. Finally, we combine the results obtained in this paper with those known in the literature to derive new verifiable conditions en- 
suring the at-point (calmness and metric subregularity) properties of solution maps to generalized equations with subdifferential fields.

Throughout the paper we mainly use standard notation and terminology of variational analysis; see, e.g., $[5,17,23]$ and Section 2 for more details. Recall that $\mathcal{L}(X, Y)$ stands for the collection of all linear bounded operators $A: X \rightarrow Y$ between Banach spaces, that $\bar{R}:=\mathbb{R} \cup\{\infty\}$ denotes the extended real line, and that $\mathbb{N}:=\{1,2, \ldots\}$ is the set of all natural numbers.

\section{Basic Definitions and Preliminaries}

This section presents basic definitions and preliminaries widely used in what follows. Unless otherwise stated, all the spaces under consideration are Banach with the generic notation $\|\cdot\|$ for their norms. If no confusion arises, the symbol $\mathbb{B}$ stands for the closed unit ball of the space in question while $\mathbb{B}_{a}(\bar{x})$ indicates the closed ball of radius $a>0$ centered at $\bar{x}$.

For a set-valued mapping $F: X \rightrightarrows Y$, we denote its graph by

$$
\operatorname{gph} F:=\{(x, y) \in X \times Y \mid y \in F(x)\}
$$

and usually use the notation $f: X \rightarrow Y$ for single-valued mappings. The symbol $F^{-1}: Y \rightrightarrows X$ stands for the inverse mapping to $F$ with $\operatorname{gph} F^{-1}=\{(y, x) \in Y \times X \mid(x, y) \in \operatorname{gph} F\}$.

Given subsets $C, D \subset X$, define the distance from $x \in X$ to $C$ and the excess from $C$ to $D$ by

$$
d(x, C):=\inf _{y \in C}\|x-y\| \text { and } e(C, D):=\sup _{x \in C} d(x, D),
$$

respectively, with the convention that

$$
d(x, \emptyset):=\infty \text { and } e(\emptyset, D):= \begin{cases}0 & \text { if } D \neq \emptyset \\ \infty & \text { otherwise }\end{cases}
$$

Recall that a single-valued mapping $f: X \times Y \rightarrow Z$ is (partially) Lipschitz continuous around $(\bar{x}, \bar{y})$ with respect to $x$ uniformly in $y$ if there are neighborhoods $U$ of $\bar{x}$ and $V$ of $\bar{y}$ along with a constant $\eta \geq 0$ such that

$$
\left\|f(x, y)-f\left(x^{\prime}, y\right)\right\| \leq \eta\left\|\dot{x}-x^{\prime}\right\| \text { whenever } x, x^{\prime} \in U \text { and } y \in V .
$$

The infimum of $\eta$ over all such combinations of $\eta, U$, and $V$ is called the (exact) partial uniform Lipschitz modulus of $f$ in $x$ around $(\bar{x}, \bar{y})$ and is denoted by $\widehat{\operatorname{lip}}_{x} f(\bar{x}, \bar{y})$.

Given $f: X \times Y \rightarrow Z$, we say that a function $h: Y \rightarrow Z$ is a strict estimator of $f$ around $(\bar{x}, \bar{y})$ with respect to $y$ uniformly in $x$ with constant $\lambda \geq 0$ if

$$
h(\bar{y})=f(\bar{x}, \bar{y}) \text { and } \widehat{\operatorname{lip}}_{y} g(\bar{x}, \bar{y}) \leq \lambda<\infty \text { for } g(x, y):=f(x, y)-h(y) .
$$

The following contraction principle held in complete metric spaces (see, e.g., [5, Theorem 5E.2] and the references therein) is used in some proofs of this paper in the Banach space setting.

Theorem 2.1. (Contraction principle for set-valued mappings.) Let $\Phi: X \rightrightarrows X$ be a setvalued mapping, let $\bar{x} \in X$, and let $a>0$ be such that the set $\operatorname{gph} \Phi \cap\left(\mathbb{B}_{a}(\bar{x}) \times \mathbb{B}_{a}(\bar{x})\right)$ is closed in $X \times X$. Given $\theta \in(0,1)$, impose the following assumptions:

(i) $d(\bar{x}, \Phi(\bar{x}))<a(1-\theta)$;

(ii) $e\left(\Phi(u) \cap \mathbb{B}_{a}(\bar{x}), \Phi(v)\right) \leq \theta\|u-v\|$ for all $u, v \in \mathbb{B}_{a}(\bar{x})$.

Then $\Phi$ has a fixed point in $\mathbb{B}_{a}(\bar{x})$, i.e., there is $x \in \mathbb{B}_{a}(\bar{x})$ with $x \in \Phi(x)$. 
Next we define the major metric regularity and Lipschitzian properties of our study.

Definition 2.2. (Metric regularity and subregularity.) Given a set-valued mapping $F: X \rightrightarrows Y$ and a point $(\bar{x}, \bar{y}) \in \operatorname{gph} F$, we say that:

(i) $F$ is METRICALLY REGULAR AROUND $(\bar{x}, \bar{y})$ with constant/modulus $\kappa>0$ if there are neighborhoods $U \subset X$ and $V \subset Y$ such that

$$
d\left(x, F^{-1}(y)\right) \leq \kappa d(y, F(x)) \text { for all } x \in U \text { and } y \in V .
$$

The infimum of $\kappa>0$ over all the combinations $(\kappa, U, V)$ for which (2.4) holds is called the EXACT REGULARITY BOUND of $F$ around $(\bar{x}, \bar{y})$ and is denoted by reg $F(\bar{x}, \bar{y})$.

(ii) $F$ is METRICALLY REgUlAR AT $(\bar{x}, \bar{y})$ (or SUBREGULAR at this point) with constant $\kappa>0$ if there is a neighborhood $U \subset X$ such that

$$
d\left(x, F^{-1}(\bar{y})\right) \leq \kappa d(\bar{y}, F(x)) \text { for all } x \in U .
$$

The infimum of $\kappa>0$ over all the combinations $(\kappa, U)$ for which (2.5) holds is called the EXACT SUBREGULARITY BOUND of $F$ at $(\bar{x}, \bar{y})$ and is denoted by subreg $F(\bar{x}, \bar{y})$.

It is easy to check (see, e.g., [17, Proposition 1.48]) that conditions (2.4) and (2.5) can be equivalently rewritten as

$$
d\left(x, F^{-1}(y)\right) \leq \kappa d(y, F(x) \cap \widetilde{V}) \text { and } d\left(x, F^{-1}(\bar{y})\right) \leq \kappa d(\bar{y}, F(x) \cap \widetilde{V}) \text { for all } x \in \widetilde{U},
$$

where $\widetilde{U} \subset X$ and $\widetilde{V} \subset Y$ are some neighborhoods of $\bar{x}$ and $\bar{y}$, respectively, and where the first estimate in (2.6) is required to hold for all $y \in Y$ sufficiently close to $\bar{y}$.

As we can clearly see from (2.6), the difference between the metric regularity properties of Definition 2.2 at and around the given point $(\vec{x}, \bar{y})$ is that the underlying distance estimate in (ii) is taken at the fixed point $\bar{y}$ in question while the corresponding estimate in (i) is required for all $y$ close to $\bar{y}$ with the uniform modulus $\kappa>0$. In the classical settings both these properties were implicitly used (in some equivalent forms) by Lyusternik [14] and Graves [7]. In the commentaries to $[5,9,12,17]$ the reader can find comprehensive information on the evolution of these properties and their various applications with more references and discussions.

By now it has been well recognized that the "at" and "around" properties of metric regularity from Definition 2.2 are significantly different from each other. This was strongly emphasized by Milyutin, long before publishing his joint paper [4], who called (2.4) and its modifications by properties in a neighborhood in contrast to their "one-point" counterparts as, e.g., in Ioffe [8], where the metric (sub)regularity property of the latter type was introduced and developed in the case of nonsmooth Lipschitzian mappings $f: X \rightarrow Y$ in (2.5). It turns out that robustness of metric regularity (2.4) around $(\bar{x}, \bar{y})$, besides its linear rate, allows us to obtain complete characterizations of this and related neighborhood properties with precise formulas for computing the exact regularity bound $\operatorname{reg} F(\bar{x}, \bar{y})$ in terms of pointwise coderivative constructions satisfying full calculus; see [15, 17, 23]. This does not seem to be possible for the unstable at-point/subregularity version from Definition 2.2(ii). Observe to this end that the zero function from $\mathbb{R}$ to $\mathbb{R}$ is surely subregular at $\bar{x}=0$ while this property vanishes after adding the function $g(x)=x^{2}$. Other examples and discussions in this direction can be found in [5, pp. 184-185] and in Remarks 3.4, 3.6, 5.5 presented below. In what follows we try to avoid (as in $[4,9,17]$ and many other publications) using the "at-point" terminology adopted in $[5,23]$ for neighborhood properties of type $(2.4)$, which can lead to confusion according to our understanding and experience.

The discussion above equally applies also to the following properties of the Lipschitzian type for set-valued mappings "at" and "around" the points in questions; see more details in $[5,9,17,23]$. 
Definition 2.3. (Lipschitzian properties of set-valued mappings.) Given $F: X \rightrightarrows Y$ and $(\bar{x}, \bar{y}) \in \operatorname{gph} F$, we say that:

(i) $F$ is LIPSCHITZ-LIKE AROUND $(\bar{x}, \bar{y})$, or has the AUBIN PROPERTY around this point, with modulus $\ell \geq 0$ if there are neighborhoods $U$ of $\bar{x}$ and $V$ of $\bar{y}$ such that

$$
F(x) \cap V \subset F\left(x^{\prime}\right)+\ell\left\|x-x^{\prime}\right\| \mathbb{B} \text { for all } x, x^{\prime} \in U
$$

The infimum of $\ell \geq 0$ over all the combinations $(\ell, U, V)$ for which $(2.7)$ holds is called the EXACT LIPSCHITZIAN BOUND of $F$ around $(\bar{x}, \bar{y})$ and is denoted by $\operatorname{lip} F(\bar{x}, \bar{y})$.

(ii) $F$ is CALM AT $(\bar{x}, \bar{y})$ with modulus $\ell$ if there are neighborhoods $U$ of $\bar{x}$ and $V$ of $\bar{y}$ such that

$$
F(x) \cap V \subset F(\bar{x})+\ell\|x-\bar{x}\| \mathbb{B} \text { for all } x \in U .
$$

The infimum of $\ell \geq 0$ over all the combinations $(\ell, U, V)$ for which (2.8) holds is called the EXACT BOUND OF CALMNESS for $F$ at $(\bar{x}, \bar{y})$ and is denoted by $\operatorname{clm} F(\bar{x}, \bar{y})$.

It is easy to observe, under the local closedness of $F(x)$ around the reference point, that inclusions (2.7) and (2.8) can be equivalently written via the corresponding excess (2.1) as, respectively,

$$
e\left(F(x) \cap V, F\left(x^{\prime}\right)\right) \leq \ell\left\|x-x^{\prime}\right\| \text { and } e(F(x) \cap V, F(\bar{x})) \leq \ell\|x-\bar{x}\| \text { for all } x, x^{\prime} \in U .
$$

Similarly to the metric regularity/subregularity properties from Definition 2.2 we have furthermore that conditions (2.9) can be in turn equivalently written as

$e\left(F(x) \cap \tilde{V}, F\left(x^{\prime}\right)\right) \leq \ell\left\|x-x^{\prime}\right\|$ and $e(F(x) \cap \widetilde{V}, F(\bar{x})) \leq \ell\|x-\bar{x}\|$ for all $x \in X$ and $x^{\prime} \in \tilde{U}$,

where $\widetilde{U} \subset X$ and $\tilde{V} \subset Y$ are appropriate neighborhoods of $\bar{x}$ and $\bar{y}$.

The Lipschitz-like property from Definition 2.3(i) was introduced by Aubin [2] as the "pseudoLipschitz" property. It reduces to the classical (Hausdorff) local Lipschitz continuity of $F$ around $\bar{x}$ if $V=Y$ in (2.7) and surely gives back the classical local Lipschitzian property for single-valued mappings. It seems that the Lipschitz-like/Aubin property (2.7) is the most natural extension of the classical local Lipschitz continuity to set-valued mappings being a graphical counterpart of the latter in the set-valued case; see $[17,23]$ for more discussions.

In contrast, the calmness property from Definition 2.3(ii) does not go back to the classical local Lipschitz continuity in the case of single-valued mappings, which requires comparison between two points in a neighborhood of the reference one, while in (2.8) we have $x^{\prime}=\bar{x}$ fixed. When $V=Y$ in (2.8), this property was introduced by Robinson [21] as the "upper Lipschitz" property of set-valued and single-valued mappings. The graphical localization in (2.8) and the "calmness" terminology in this framework appeared in [23]. In $[5,10,12,25]$, the reader can find more information and discussion on the calmness property with recent results and applications.

It has been long recognized in variational analysis the equivalence between appropriate metric regularity properties of mappings and Lipschitzian properties of their inverses. The proofs of such results are straightforward and more or less based just on the definitions; see, e.g., [17, Theorem 1.49] and [5, Theorem 3H.3] for the corresponding neighborhood and one-point properties from Definitions 2.2 and 2.3 with the commentaries therein.

Proposition 2.4. (Equivalence between metric regularity and Lipschitzian properties.) For an arbitrary mapping $F: X \rightrightarrows Y$ with $(\bar{x}, \bar{y}) \in \operatorname{gph} F$, the following assertions hold: 
(i) $F$ is metrically regular around $(\bar{x}, \bar{y})$ if and only if the inverse mapping $F^{-1}: Y \rightrightarrows X$ is Lipschitz-like around $(\bar{y}, \bar{x})$. Furthermore, we have the equality

$$
\operatorname{reg} F(\bar{x}, \bar{y})=\operatorname{lip} F^{-1}(\bar{y}, \bar{x}) \text {. }
$$

(ii) $F$ is metrically subregular at $(\bar{x}, \bar{y})$ if and only if the inverse mapping $F^{-1}: Y \rightrightarrows X$ is calm at $(\bar{y}, \bar{x})$. Furthermore, we have the equality

$$
\operatorname{subreg} F(\bar{x}, \bar{y})=\operatorname{clm} F^{-1}(\bar{y}, \bar{x}) .
$$

As mentioned in the above discussion on metric regularity, this property around the points in questions admits full pointwise characterizations with computing the exact bound of moduli in (2.4) in the general setting of set-valued mappings. In this paper we need the corresponding characterization and formula only for the case of linear operators between Banach spaces from [17, Corollary 1.58], which is in fact a refined version of the classical Banach-Schauder open mapping theorem directed originally (as well as its nonlinear Lyusternik-Graves extension) to deriving sufficient conditions for openness/metric regularity with no modulus estimates.

Proposition 2.5. (Metric regularity for linear bounded operators.) A linear bounded operator $A \in \mathcal{L}(X, Y)$ is metrically regular around every point $x \in X$ if and only if it is surjective. In this case the exact regularity bound of $A$ is computed by

$$
\operatorname{reg} A=\left\|\left(A^{*}\right)^{-1}\right\|
$$

via the norm of the (single-valued) inverse to the adjoint operator $A^{*} \in \mathcal{L}\left(Y^{*}, X^{*}\right)$.

\section{Metric Regularity of Solution Maps via Lipschitzian Properties of Fields in Generalized Equations}

The primary goal of this section is to find appropriate conditions imposed on the initial data of the generalized equation (1.1) that ensure the equivalence between the metric regularity of the solution map $S: X \rightrightarrows Y$ from (1.2) around $(\bar{x}, \bar{y})$ and the Lipschitz-like property of the field mapping $Q: Y \rightrightarrows Z$ of $(1.1)$ around $(\bar{y},-f(\bar{x}, \bar{y}))$, with establishing relationships between the corresponding exact bounds. Furthermore, we derive similar results relating the at-point metric subregularity and calmness properties of $S$ and $Q$, respectively.

The following lemma concerning the base mapping $f$ in (1.1) plays a significant role in our consideration. Its proof is a certain modification of the Lyusternik-Graves iterative process (cf. [7, 14]) while the result itself is a far-going extension of [7, Theorem 1] providing also a tight relationship between the exact bounds of the corresponding moduli; see more discussions in Remark 3.2 below.

Lemma 3.1. (Implicit multifunctions.) Let $f: X \times Y \rightarrow Z$ be a mapping between Banach spaces, and let $(\bar{x}, \bar{y}) \in X \times Y$ be such that $f$ is locally Lipschitzian with respect to $y$ with constant $\eta \geq 0$ uniformly in $x$ on some neighborhood $U \times V$ of $(\bar{x}, \bar{y})$. Given a surjective linear operator $A \in \mathcal{L}(X, Z)$, suppose that there are $\mu \geq 0$ and $\gamma>\operatorname{reg} A$ satisfying the relationships $\mu \gamma<1$ and

$$
\left\|f(x, y)-f\left(x^{\prime}, y\right)-A\left(x-x^{\prime}\right)\right\| \leq \mu\left\|x-x^{\prime}\right\| \text { for all } x, x^{\prime} \in U \text { and } y \in V .
$$

Given further a mapping $g: W \rightarrow Z$ between Banach spaces that is locally Lipschitzian around $\bar{w} \in W$ with constant $\lambda$, consider a set-valued mapping $\Gamma: Y \times W \rightrightarrows X$ defined by

$$
\Gamma(y, w):=\{x \in X \mid f(x, y)+g(w)=0\} .
$$


Then there is $\alpha>0$ such that for every $(y, w),\left(y^{\prime}, w^{\prime}\right) \in \mathbb{B}_{\alpha}(\bar{y}) \times \mathbb{B}_{\alpha}(\bar{w})$ we have the inclusion

$$
\Gamma\left(y^{\prime}, w^{\prime}\right) \cap \mathbb{B}_{\alpha}(\bar{x}) \subset \Gamma(y, w)+\frac{\gamma}{1-\gamma \mu}\left(\eta\left\|y-y^{\prime}\right\|+\lambda\left\|w-w^{\prime}\right\|\right) \mathbb{B} .
$$

The latter implies, when $g(\bar{w})=-f(\bar{x}, \bar{y})$, that $\Gamma$ is Lipschitz-like around $((\bar{y}, \bar{w}), \bar{x})$ with the following upper estimate of the exact Lipschitzian bound:

$$
\operatorname{lip} \Gamma((\bar{y}, \bar{w}), \bar{x}) \leq \frac{\operatorname{reg} A \cdot \max \left\{\widehat{\operatorname{lip}}_{y} f(\bar{x}, \bar{y}), \operatorname{lip} g(\bar{w})\right\}}{1-\mu \cdot \operatorname{reg} A} .
$$

Proof. The Lipschitz-like property of $\Gamma$ around $((\bar{y}, \bar{w}), \bar{x})$ and the exact bound estimate (3.4) follow directly from (3.3), Definition 2.3(i) and the assumptions of the theorem. To justify (3.3), pick $a>0$ such that (3.1) holds for every $x, x^{\prime} \in \mathbb{B}_{a}(\bar{x})$ and $y \in \mathbb{B}_{a}(\bar{y})$, that $f$ is Lipschitz continuous with respect to $y$ uniformly in $x$ on $\mathbb{B}_{a}(\bar{x}) \times \mathbb{B}_{a}(\bar{y})$ with constant $\eta \geq 0$, and that $g$ is Lipschitz continuous on $\mathbb{B}_{a}(\bar{w})$. Choose further $0<\alpha \leq a$ satisfying the estimates

$$
\alpha(2 \gamma(\lambda+\eta)+1) \leq a \text { and }\left(\frac{2 \gamma(\lambda+\eta)}{1-\gamma \mu}+1\right) \alpha \leq a
$$

Fix arbitrary pairs $(y, w),\left(y^{\prime}, w^{\prime}\right) \in \mathbb{B}_{\alpha}(\bar{y}) \times \mathbb{B}_{\alpha}(\bar{w})$ and take some point $x^{\prime} \in \Gamma\left(y^{\prime}, w^{\prime}\right) \cap \mathbb{B}_{\alpha}(\bar{x})$ observing that we have inclusion (3.3) automatically if the latter point does not exist.

Starting with $x_{0}:=x^{\prime}$, we construct next a sequence $\left\{x_{k}\right\} \subset \mathbb{B}_{a}(\bar{x})$ by using the following iterates of the Lyusternik-Graves type as $k \in \mathbb{N}$ :

$$
\begin{aligned}
& A\left(x_{k}-x_{k-1}\right)=-g(w)-f\left(x_{k-1}, y\right) \text { with } \\
& \left\|x_{k}-x_{k-1}\right\| \leq \gamma(\gamma \mu)^{k-1}\left\|g(w)+f\left(x^{\prime}, y\right)\right\| .
\end{aligned}
$$

Let us first justify the well-posedness of the suggested iterative procedure. Indeed, by the surjectivity of the operator $A$ from (3.1) we find $x_{1} \in X$ such that

$$
A\left(x_{1}-x^{\prime}\right)=-g(w)-f\left(x^{\prime}, y\right) \text { with }\left\|x_{1}-x^{\prime}\right\| \leq \gamma\left\|g(w)+f\left(x^{\prime}, y\right)\right\| .
$$

Then the assumptions of the lemma ensure the estimates

$$
\begin{aligned}
\left\|x_{1}-\bar{x}\right\| & \leq\left\|x_{1}-x^{\prime}\right\|+\left\|x^{\prime}-\bar{x}\right\| \leq \gamma\left\|g(w)+f\left(x^{\prime}, y\right)\right\|+\alpha \\
& \leq \gamma\left\|g(w)-g\left(w^{\prime}\right)\right\|+\gamma\left\|f\left(x^{\prime}, y\right)-f\left(x^{\prime}, y^{\prime}\right)\right\|+\alpha \\
& \leq \gamma \lambda\left\|w-w^{\prime}\right\|+\gamma \eta\left\|y-y^{\prime}\right\|+\alpha \\
& \leq \alpha(2 \gamma(\lambda+\eta)+1) \leq a
\end{aligned}
$$

This implies that $x_{1} \in \mathbb{B}_{a}(\bar{x})$ and that $x_{1}$ satisfies relationships (3.6) and (3.7). Suppose now that for some $n \in \mathbb{N}$ we have constructed the iterates $x_{1}, \ldots, x_{n}$ in $\mathbb{B}_{a}(\bar{x})$ satisfying (3.6) and (3.7). Using again the surjectivity of $A$, get $x_{n+1} \in X$ such that $A\left(x_{n+1}-x_{n}\right)=-g(w)-f\left(x_{n}, y\right)$ and

$$
\begin{aligned}
\left\|x_{n+1}-x_{n}\right\| & \leq \gamma\left\|g(w)+f\left(x_{n}, y\right)\right\|=\gamma\left\|f\left(x_{n}, y\right)-f\left(x_{n-1}, y\right)-A\left(x_{n}-x_{n-1}\right)\right\| \\
& \leq \gamma \mu\left\|x_{n}-x_{n-1}\right\| \leq \gamma(\gamma \mu)^{n}\left\|g(w)+f\left(x^{\prime}, y\right)\right\| .
\end{aligned}
$$


The latter yields furthermore that

$$
\begin{aligned}
\left\|x_{n+1}-x^{\prime}\right\| & \leq \sum_{i=1}^{n+1}\left\|x_{i}-x_{i-1}\right\| \leq \gamma\left\|g(w)+f\left(x^{\prime}, y\right)\right\| \sum_{i=1}^{n+1}(\gamma \mu)^{i} \\
& \leq \frac{\gamma}{1-\gamma \mu}\left\|g(w)+f\left(x^{\prime}, y\right)\right\| \\
& \leq \frac{\gamma}{1-\gamma \mu}\left(\left\|g(w)-g\left(w^{\prime}\right)\right\|+\left\|f\left(x^{\prime}, y\right)-f\left(x^{\prime}, y^{\prime}\right)\right\|\right) \\
& \leq \frac{\gamma}{1-\gamma \mu}\left(\lambda\left\|w-w^{\prime}\right\|+\eta\left\|y-y^{\prime}\right\|\right),
\end{aligned}
$$

which allows us to conclude that

$$
\left\|x_{n+1}-\bar{x}\right\| \leq\left\|x_{n+1}-x^{\prime}\right\|+\left\|x^{\prime}-\bar{x}\right\| \leq\left(\frac{2 \gamma(\lambda+\eta)}{1-\gamma \mu}+1\right) \alpha \leq a .
$$

This justifies by induction the possibility to construct a sequence $\left\{x_{k}\right\} \subset \mathbb{B}_{a}(\bar{x})$ satisfying (3.6) and (3.7). By (3.7) we get for any $m>n \geq 1$ the estimates

$$
\begin{aligned}
\left\|x_{m}-x_{n}\right\| & \leq \sum_{i=n+1}^{m}\left\|x_{i}-x_{i-1}\right\| \leq \gamma\left\|g(w)+f\left(x^{\prime}, y\right)\right\| \sum_{i=n+1}^{m}(\gamma \mu)^{i-1} \\
& \leq \gamma(\gamma \mu)^{n}\left\|g(w)+f\left(x^{\prime}, y\right)\right\| \sum_{i=0}^{\infty}(\gamma \mu)^{i}=\frac{\gamma}{1-\gamma \mu}\left\|g(w)+f\left(x^{\prime}, y\right)\right\|(\gamma \mu)^{n}
\end{aligned}
$$

which proves that $\left\{x_{k}\right\}$ is a Cauchy sequence and it converges therefore to some $\widehat{x} \in \mathbb{B}_{\alpha}(\bar{x})$. Passing to the limit in (3.6) and (3.8) as $k \rightarrow \infty$ and $n \rightarrow \infty$, we obtain that $g(w)=-f(\widehat{x}, y)$ and that

$$
\left\|\widehat{x}-x^{\prime}\right\| \leq \frac{\gamma}{1-\gamma \mu}\left(\lambda\left\|w-w^{\prime}\right\|+\eta\left\|y-y^{\prime}\right\|\right) \text {. }
$$

Thus $\widehat{x} \in \Gamma(y, w)$, which verifies (3.3) and completes the proof of the lemma.

Remark 3.2. (Related observations.) It is worth making the following observations regarding the results and assumptions of Lemma 3.1.

(i) Let $f(x, y)=f(x)$ and $g(y)=-y$ for $x \in X$ and $y \in Y$ in the framework of Lemma 3.1. Then the multifunction $\Gamma$ in (4.5) reduces to the inverse mapping $\Gamma(y)=f^{-1}(y)$. Taking into account that the Lipschitz-like property of the inverse mapping $f^{-1}$ around $\bar{y}$ is equivalent by Proposition 2.4(i) to the metric regularity of $f$ around $\bar{x}$ while the latter is equivalent in turn to the so-called linear openness/covering property of $f$ around this point that is actually considered in [7], we get from Lemma 3.1 the main result of Graves [7]. By now it has been well recognized in variational analysis that the surjectivity condition on the (strict) derivative of $f$ similar to (3.1) fully characterizes the metric regularity and equivalent properties of nonlinear mappings around the corresponding points; see, e.g., [17, Theorem 1.57] and the commentaries therein.

(ii) The calmness version of Lemma 3.1 is also valid: under the weaker assumptions

$$
\widehat{\operatorname{clm}}_{y} f(\bar{x}, \bar{y})<\infty \text { and } \operatorname{clm} g(\bar{w})<\infty
$$

in the framework of Lemma 3.1 we get by a symmetric proof that the set-valued mapping $\Gamma$ from $(4.5)$ is calm at $((\bar{y}, \bar{w}), \bar{x})$ when $f(\bar{x}, \bar{y})=-g(\bar{w})$ with the exact bound estimate

$$
\operatorname{clm} \Gamma(\bar{y}, \bar{w}), \bar{x}) \leq \frac{\operatorname{reg} A \cdot \max \left\{\widehat{\operatorname{clm}}_{y} f(\bar{x}, \bar{y}), \operatorname{clm} g(\bar{w})\right\}}{1-\mu \cdot \operatorname{reg} A},
$$


where $\widehat{\operatorname{clm}}_{y} f(\bar{x}, \bar{y})$ is defined similarly to $\widehat{\operatorname{lip}}_{y} f(\bar{x}, \bar{y})$ as in Section 2 .

(iii) If $f: X \times Y \rightarrow Z$ is Lipschitz continuous with respect to $y$ uniformly in $x$ around $(\bar{x}, \bar{y})$ with constant $\eta \geq 0$ and if there is an operator $A \in \mathcal{L}(X, Z)$ satisfying (3.1), then $f$ is locally Lipschitzian around this point with respect to both variables. Indeed, for any $(x, y)$ and $\left(x^{\prime}, y^{\prime}\right)$ close to $(\bar{x}, \bar{y})$ we have the inequalities

$$
\begin{aligned}
\left\|f(x, y)-f\left(x^{\prime}, y^{\prime}\right)\right\| \leq & \left\|f(x, y)-f\left(x, y^{\prime}\right)\right\|+\left\|f\left(x, y^{\prime}\right)-f\left(x^{\prime}, y^{\prime}\right)-A\left(x-x^{\prime}\right)\right\| \\
& +\|A\| \cdot\left\|x-x^{\prime}\right\| \\
\leq & \eta\left\|y-y^{\prime}\right\|+(\mu+\|A\|)\left\|x-x^{\prime}\right\|,
\end{aligned}
$$

which justify the local Lipschitz continuity of $f$ with respect to both $x$ and $y$ around $(\bar{x}, \bar{y})$.

As discussed in Section 2, the at-point properties of subregularity and calmness are essentially different in general from (and less developed than) their metric regularity and Lipschitz-like counterparts defined around the reference points. However, in what follows we discover some important settings, where the aforementioned "at" and "around" properties behave in a similar way. Furthermore, the proofs of these results are fully symmetric and can be unified. To proceed in this direction, we rewrite the underlying metric regularity and subregularity inequalities (2.4) and (2.5) in the unified way

$$
d\left(x, F^{-1}(\bar{y})\right) \leq \kappa d(y, F(x)) \text { for all } x \in \mathbb{B}_{a}(\bar{x}) \text { and } y \in \mathbb{B}_{b}(\bar{y})
$$

where the constants $a$ and $\kappa$ are positive while $b \geq 0$. The latter allows us to include into framework (3.9) both metric regularity property of $F$ around $(\bar{x}, \bar{y}) \in \operatorname{gph} F$ for $b>0$ and metric subregularity property of $F$ at $(\bar{x}, \bar{y})$ corresponding to $b=0$ with the convention that $\mathbb{B}_{0}(\bar{y})=\{\bar{y}\}$ in (3.9). Similarly we unify the underlying Lipschitz-like and calmness inclusions (2.7) and (2.8) as

$$
F(x) \cap \mathbb{B}_{a}(y) \subset F(u)+\ell\|x-u\| \mathbb{B} \text { for all } x \in \mathbb{B}_{a}(\bar{x}) \text { and } u \in \mathbb{B}_{b}(\bar{x})
$$

with $\ell \geq 0, a>0$, and $b \geq 0$, where the case of $b>0$ in (3.10) corresponds to the Lipschitz-like property of $F$ around $(\bar{x}, \bar{y})$, while $b=0$ therein gives us the calmness property of $F$ at $(\bar{x}, \bar{y})$.

The next theorem establishes the equivalence, on one hand, between metric regularity of the solution map $S$ in (1.2) and the Lipschitz-like property of the field $Q$ in (1.1) around the reference points and, on the other hand, between subregularity of $S$ and calmness of $Q$ at these points. Furthermore, we derive tight quantitative relationships between the exact bounds of moduli for the corresponding properties via the initial data of (1.1).

Theorem 3.3. (Metric regularity and subregularity of solution maps via Lipschitzian properties of fields in generalized equations.) Let $f: X \times Y \rightarrow Z$ be a mapping between Banach spaces that is Lipschitz continuous on a neighborhood $U \times V$ of $(\bar{x}, \bar{y}) \in X \times Y$, and let $Q: Y \rightrightarrows Z$ be a set-valued field mapping with $\bar{z}:=-f(\bar{x}, \bar{y}) \in Q(\bar{y})$. Assume that $A \in \mathcal{L}(X, Z)$ is a surjective linear operator such that there is $\mu \geq 0$ with

$$
\mu \cdot \operatorname{reg} A<1 \text { and }\left\|f(x, y)-f\left(x^{\prime}, y\right)-A\left(x-x^{\prime}\right)\right\| \leq \mu\left\|x-x^{\prime}\right\| \text { for all } x, x^{\prime} \in U, y \in V \text {. }
$$

Then the following assertions are satisfied:

(i) The solution map $S$ in (1.2) is metrically regular around $(\bar{x}, \bar{y})$ if and only if the field $Q$ in (1.1) is Lipschitz-like around $(\bar{y}, \bar{z})$. Moreover, we have the exact bound relationships

$$
\begin{array}{r}
\operatorname{reg} S(\bar{x}, \bar{y}) \leq \frac{\operatorname{reg} A \cdot\left[\operatorname{lip} Q(\bar{y}, \bar{z})+\widehat{\operatorname{lip}}_{y} f(\bar{x}, \bar{y})\right]}{1-\mu \cdot \operatorname{reg} A}, \\
\operatorname{lip} Q(\bar{y}, \bar{z}) \leq \widehat{\operatorname{lip}}_{x} f(\bar{x}, \bar{y}) \cdot \operatorname{reg} S(\bar{x}, \bar{y})+\widehat{\operatorname{lip}}_{y} f(\bar{x}, \bar{y}) .
\end{array}
$$


(ii) The solution map $S$ is subregular at $(\bar{x}, \bar{y})$ if and only if the field $Q$ is calm at $(\bar{y}, \bar{z})$. Furthermore, we have the exact bound relationships

$$
\begin{gathered}
\text { subreg } S(\bar{x}, \bar{y}) \leq \frac{\operatorname{reg} A \cdot\left[\operatorname{clm} Q(\bar{y}, \bar{z})+\widehat{\operatorname{lip}}_{y} f(\bar{x}, \bar{y})\right]}{1-\mu \cdot \operatorname{reg} A}, \\
\operatorname{clm} Q(\bar{y}, \bar{z}) \leq \widehat{\operatorname{lip}}_{x} f(\bar{x}, \bar{y}) \cdot \operatorname{subreg} S(\bar{x}, \bar{y})+\widehat{\operatorname{lip}}_{y} f(\bar{x}, \bar{y}) .
\end{gathered}
$$

Proof. Observe first that the general assumptions made in this theorem ensure the fulfillment of all the requirements of Lemma 3.1 with $W=Z$ and $g(z)=z$. Thus for any $\eta_{y}>\widehat{\operatorname{lip}}_{y} f(\bar{x}, \bar{y})$ and $\gamma>\operatorname{reg} A$ with $\gamma \mu<1$ there is a positive constant $\alpha$ such that for every $(y, z),\left(y^{\prime}, z^{\prime}\right) \in \mathbb{B}_{\alpha}(\bar{y}) \times \mathbb{B}_{\alpha}(\bar{z})$ we have the inclusion

$$
\Gamma\left(y^{\prime}, z^{\prime}\right) \cap \mathbb{B}_{\alpha}(\bar{x}) \subset \Gamma(y, z)+\frac{\gamma}{1-\gamma \mu}\left(\eta_{y}\left\|y-y^{\prime}\right\|+\left\|z-z^{\prime}\right\|\right) \mathbb{B},
$$

where $\Gamma(y, z):=\{x \in X \mid f(x, y)+z=0\}$.

Starting with the proof of the "only if" part in assertions (i) and (ii) simultaneously, assume that the underlying estimate (3.9) holds for the solution map $S$ on (1.2) with positive constants $\kappa, a$ and with $b \geq 0$. Take $\eta_{x}>\widehat{\operatorname{lip}}_{x} f(\bar{x}, \bar{y})$ and make $a>0$ smaller if necessary to get

$$
\begin{gathered}
d\left(x, S^{-1}(y)\right) \leq \kappa d(y, S(x)) \text { for every }(x, y) \in \mathbb{B}_{a}(\bar{x}) \times \mathbb{B}_{b}(\bar{y}) \text { and } \\
\left\|f(x, y)-f\left(x^{\prime}, y^{\prime}\right)\right\| \leq \eta_{x}\left\|x-x^{\prime}\right\|+\eta_{y}\left\|y-y^{\prime}\right\| \text { for all }(x, y),\left(x^{\prime}, y^{\prime}\right) \in \mathbb{B}_{a}(\bar{x}) \times \mathbb{B}_{a}(\bar{y})
\end{gathered}
$$

Next decrease $\alpha>0$ if necessary and choose $\beta \geq 0$ (with $\beta>0$ of $b \neq 0$ ) satisfying the relationships

$$
\alpha \leq a, \quad \beta \leq \min \{b, \alpha\}, \quad(\alpha+\beta) \kappa<\frac{a}{2}, \quad \text { and } \frac{\gamma\left(\eta_{y}+1\right)}{1-\gamma \mu} \alpha \leq \frac{a}{2} .
$$

Pick further $y \in \mathbb{B}_{\alpha}(\bar{y}), y^{\prime} \in \mathbb{B}_{\beta}(\bar{y})$, and $z \in Q(y) \cap \mathbb{B}_{\alpha}(\bar{z})$ observing that we are done if no such $z$ exists. Note also that $y^{\prime}=\bar{y}$ if $b=0$ and thus $\beta=0$. Then by (3.16) we find $x \in \Gamma(y, z)$ such that

$$
\|x-\bar{x}\| \leq \frac{\gamma}{1-\gamma \mu}\left(\eta_{y}\|y-\bar{y}\|+\|z-\bar{z}\|\right) \leq \frac{\gamma\left(\eta_{y}+1\right)}{1-\gamma \mu} \alpha \leq \frac{a}{2}
$$

Hence $y \in S(x)$ by the choice of $y$ and $z$, which allows us to conclude from the assumed metric regularity/subregularity of $S$ in (3.9) that

$$
d\left(x, S^{-1}\left(y^{\prime}\right)\right) \leq \kappa d\left(y^{\prime}, S(x)\right) \leq \kappa\left\|y^{\prime}-y\right\| .
$$

The latter implies that for every $\varepsilon>0$ there is $x_{\varepsilon} \in S^{-1}\left(y^{\prime}\right)$ with $\left\|x-x_{\varepsilon}\right\| \leq \kappa\left\|y^{\prime}-y\right\|+\varepsilon$, and so

$$
\left\|x_{\varepsilon}-\bar{x}\right\| \leq\left\|x_{\varepsilon}-x\right\|+\|x-\bar{x}\| \leq(\alpha+\beta) \kappa+\varepsilon+a / 2 \leq a
$$

whenever $\varepsilon>0$ is sufficiently small. Letting now $z_{\varepsilon}:=-f\left(x_{\varepsilon}, y^{\prime}\right)$, we get $z_{\varepsilon} \in Q\left(y^{\prime}\right)$ satisfying

$$
\left\|z-z_{\varepsilon}\right\|=\left\|f(x, y)-f\left(x_{\varepsilon}, y^{\prime}\right)\right\| \leq \eta_{x}\left\|x-x_{\varepsilon}\right\|+\eta_{y}\left\|y-y^{\prime}\right\| \leq\left(\kappa \eta_{x}+\eta_{y}\right)\left\|y-y^{\prime}\right\|+\eta_{x} \varepsilon,
$$

which gives $d\left(z, Q\left(y^{\prime}\right)\right) \leq\left(\kappa \eta_{x}+\eta_{y}\right)\left\|y-y^{\prime}\right\|+\eta_{x} \varepsilon$. Passing to the limit in the latter expression as $\varepsilon \downarrow 0$ allows us to conclude that

$$
d\left(z, Q\left(y^{\prime}\right)\right) \leq\left(\kappa \eta_{x}+\eta_{y}\right)\left\|y-y^{\prime}\right\| \text { for all } z \in Q(y) \cap \mathbb{B}_{\alpha}(\bar{z})
$$


and therefore $Q$ is Lipschitz-like around $(\bar{y}, \bar{z})$ with $\operatorname{lip} Q(\bar{y}, \bar{z}) \leq \kappa \eta_{x}+\eta_{y}$ when $b>0$, and it is calm at this point with $\operatorname{clm} Q(\bar{y}, \bar{z}) \leq \kappa \eta_{x}+\eta_{y}$ if $b=0$. Since the constants $\eta_{x}$ and $\eta_{y}$ above can be chosen arbitrarily close to $\widehat{\operatorname{lip}}_{x} f(\bar{x}, \bar{y})$ and $\widehat{\operatorname{lip}}_{y} f(\bar{x}, \bar{y})$, respectively, while $\kappa$ is arbitrarily close to reg $S(\bar{x}, \bar{y})$ for $b>0$ and to subreg $S(\bar{x}, \bar{y})$ for $b=0$, we arrive at the corresponding exact bound estimates (3.13) and (3.15) and thus complete the proof of the "only if" part of the theorem in both assertions (i) and (ii).

To justify next the "if" part in assertions (i) and (ii) simultaneously, assume that inclusion (3.10) holds for the field $Q$, i.e., we have

$$
Q(y) \cap \mathbb{B}_{a}(\bar{z}) \subset Q\left(y^{\prime}\right)+\ell\left\|y-y^{\prime}\right\| \mathbb{B} \text { whenever } y \in \mathbb{B}_{a}(\bar{y}) \text { and } y^{\prime} \in \mathbb{B}_{b}(\bar{y})
$$

with some constants $\ell \geq 0, a>0$, and $b \geq 0$, where $b>0$ corresponds to the Lipschitz-like property of $Q$ around $(\bar{y}, \bar{z})$ while $b=0$ relates to the calmness property of $Q$ at this point. Pick any $\eta_{x}>\widehat{\operatorname{lip}}_{x} f(\bar{x}, \bar{y})$ and make $a$ smaller if necessary so that

$$
\left\|f(x, y)-f\left(x^{\prime}, y^{\prime}\right)\right\| \leq \eta_{x}\left\|x-x^{\prime}\right\|+\eta_{y}\left\|y-y^{\prime}\right\| \text { for all }(x, y),\left(x^{\prime}, y^{\prime}\right) \in \mathbb{B}_{a}(\bar{x}) \times \mathbb{B}_{a}(\bar{y}) .
$$

Take $\alpha>0$ in (3.16) with $\alpha \leq a$ and select some constants $\gamma>0$ and $\beta \geq 0$ (with $\beta>0$ if $b \neq 0$ ) satisfying the inequalities

$$
\gamma \leq \alpha, \quad \beta \leq \min \{\alpha, b\}, \quad 2 \gamma\left(\eta_{x}+\eta_{y}\right) \leq \alpha, \text { and } 2 \ell(\gamma+\beta) \leq \alpha .
$$

Fix further $y \in \mathbb{B}_{\gamma}(\bar{y}), y^{\prime} \in \mathbb{B}_{\beta}(\bar{y})$, and $x \in S^{-1}(y) \cap \mathbb{B}_{\gamma}(\bar{x})$ observing that there is nothing to prove if such a point $x$ does not exist. Then for $z:=-f(x, y)$ we have $z \in Q(y)$ and

$$
\|z-\bar{z}\| \leq \eta_{x}\|x-\bar{x}\|+\eta_{y}\|y-\bar{y}\| \leq\left(\eta_{x}+\eta_{y}\right) \gamma \leq \alpha / 2 \leq a .
$$

Thus $z \in Q(y) \cap \mathbb{B}_{a}(\bar{z})$, and by (3.17) there is $z^{\prime} \in Q\left(y^{\prime}\right)$ satisfying $\left\|z^{\prime}-z\right\| \leq \ell\left\|y^{\prime}-y\right\|$. Then

$$
\left\|z^{\prime}-\bar{z}\right\| \leq\left\|z^{\prime}-z\right\|+\|z-\bar{z}\| \leq \ell\left\|y^{\prime}-y\right\|+\alpha / 2 \leq \ell(\gamma+\beta)+\alpha / 2 \leq \alpha,
$$

which gives $z, z^{\prime} \in \mathbb{B}_{\alpha}(\bar{z})$. Since $y, y^{\prime} \in \mathbb{B}_{\alpha}(\bar{y})$, we obtain from (3.16) that

$$
x \in \Gamma(y, z) \subset \Gamma\left(y^{\prime}, z^{\prime}\right)+\frac{\gamma}{1-\gamma \mu}\left(\eta_{y}\left\|y-y^{\prime}\right\|+\left\|z-z^{\prime}\right\|\right) \mathbb{B}
$$

ensuring the existence of $x^{\prime} \in \Gamma\left(y^{\prime}, z^{\prime}\right)$ with $\left\|x-x^{\prime}\right\| \leq \gamma /(1-\gamma \mu)\left(\eta_{y}\left\|y-y^{\prime}\right\|+\left\|z-z^{\prime}\right\|\right)$. Then $-f\left(x^{\prime}, y^{\prime}\right)=z^{\prime} \in Q\left(y^{\prime}\right)$ or, equivalently, $x^{\prime} \in S^{-1}\left(y^{\prime}\right)$. Even more, we get

$$
\left\|x-x^{\prime}\right\| \leq \frac{\gamma}{1-\gamma \mu}\left(\eta_{y}\left\|y-y^{\prime}\right\|+\left\|z-z^{\prime}\right\|\right) \leq \frac{\gamma\left(\eta_{y}+\ell\right)}{1-\gamma \mu}\left\|y-y^{\prime}\right\|
$$

Since $\gamma$ and $\eta_{y}$ can be chosen arbitrarily close to $\operatorname{reg} A$ and $\widehat{\operatorname{lip}}_{y} f(\bar{x}, \bar{y})$, respectively, while $\ell$ can be arbitrarily close to $\operatorname{lip} Q(\bar{y}, \bar{z})$ for $b>0$ and to $\operatorname{clm} Q(\bar{y}, \bar{z})$ for $b=0$, the last estimate in (3.18) implies the exact bound formulas in (3.12) and (3.14) and completes the proof of the theorem.

Remark 3.4. (Discussion on assumptions and conclusions of Theorem 3.3.) We have the following observations and examples concerning the assumptions and results obtained above.

(i) Note first that the surjectivity requirement on the linear bounded operator $A$ is essential for the validity of the equivalence between the metric regularity and Lipschitz-like properties in Theorem 3.3. Indeed, for $f \equiv 0$ it is easy to observe that the solution map $S$ is metrically regular 
around $(\bar{x}, \bar{y})$ if and only if $\bar{y} \in \operatorname{int} Q^{-1}(0)$. However, the latter condition is not equivalent in general to the Lipschitz-like property of the field $Q$ around $(\bar{y}, 0)$. To illustrate this, consider mappings $Q_{1}: Y \rightrightarrows Z$ and $Q_{2}: Y \rightrightarrows Y$ defined by

$$
Q_{1}(y):=\left\{\begin{array}{ll}
\{0\} & \text { for } y=0 \\
Z & \text { for } y \neq 0
\end{array}\right\} \quad \text { and } \quad Q_{2}(y):=\{y\}, \quad y \in Y .
$$

We can see that $Q_{1}$ is not Lipschitz-like around $(0,0)$ while $0 \in \operatorname{int} Q_{1}^{-1}(0)=Y$. On the other hand, $Q_{2}$ is Lipschitz-like around every point of its graph while $S$ is not metrically regular anywhere.

(ii) The upper estimates in (3.12) and (3.13) obtained by Theorem 3.3 cannot be improved without additional assumptions, in the sense that for some mappings we reach the equalities therein. To illustrate this, consider real functions $f: \mathbb{R} \times \mathbb{R} \rightarrow \mathbb{R}$ and $Q: \mathbb{R} \rightarrow \mathbb{R}$ defined by

$$
f(x, y):=a x+b y \text { and } Q(y):=c y
$$

with $a, b, c \in \mathbb{R} \backslash\{0\}$. Since we easily get

$$
\operatorname{lip} Q=|c| \quad \text { and } \quad \operatorname{reg} S=\frac{|b+c|}{|a|},
$$

the upper estimate (3.12) is satisfied as equality when $|b+c|=|c|-|b|$. Moreover, the equality holds in (3.13) when $|b+c|=|b|+|c|$.

(iii) Observe further that for $f \equiv 0$ the solution map $S$ is always metrically subregular at any point of its graph. However, this does not imply the calmness property of the field $Q$; it is illustrated by the mapping $Q_{1}$ in Remark 3.4(i). In fact, $S$ is always metrically subregular when the base mapping $f$ does not depend on the parameter $x$. On the other hand, the examples in Remark 3.4(ii) illustrate also the tightness of the upper estimates obtained in (3.14) and (3.15).

Let us now present consequences of Theorem 3.3 in the case of strict differentiability of base mappings in generalized equations. Recall that a mapping $f: X \times Y \rightarrow Z$ is strictly partially differentiable at $(\bar{x}, \bar{y})$ with respect to $x$ uniformly in $y$ with the partial derivative $\nabla_{x} f(\bar{x}, \bar{y})$ if

$$
\lim _{\substack{x \rightarrow \bar{x} \\ x^{\prime} \rightarrow \bar{x}}} \frac{f(x, y)-f\left(x^{\prime}, y\right)-\left\langle\nabla_{x} f(\bar{x}, \bar{y}), x-x^{\prime}\right\rangle}{\left\|x-x^{\prime}\right\|}=0 \text { for all } y \in Y \text { near } \bar{y} .
$$

We obviously have that the partial strict differentiability postulated in (3.19) is implied by the (full) strict differentiability of $f$ at $(\bar{x}, \bar{y})$, which ensures the local Lipschitzian property of $f$ around $(\bar{x}, \bar{y})$ and is in turn satisfied when $f$ is $C^{1}$ around this point.

Corollary 3.5. (Equivalence between metric regularity of solutions maps and Lipschitzian properties of fields for generalized equations with strictly differentiable bases.) Let $f: X \times Y \rightarrow Z$ be a mapping between Banach spaces that is locally Lipschitzian around $(\bar{x}, \bar{y})$ and strictly partially differentiable at this point with respect to $x$ uniformly in $y$ with the surjective partial derivative $\nabla_{x} f(\bar{x}, \bar{y})$, and let $Q: Y \rightrightarrows Z$ be a set-valued mapping with $\bar{z}:=-f(\bar{x}, \bar{y}) \in Q(\bar{y})$. Then the following assertions hold:

(i) The solution map $S: X \rightrightarrows Y$ in (1.2) is metrically regular around $(\bar{x}, \bar{y})$ if and only if $Q$ is Lipschitz-like around $(\bar{y}, \bar{z})$ with the upper estimates of the corresponding exact bounds:

$$
\begin{aligned}
& \operatorname{reg} S(\bar{x}, \bar{y}) \leq\left\|\left(\nabla_{x} f(\bar{x}, \bar{y})^{*}\right)^{-1}\right\| \cdot\left[\operatorname{lip} Q(\bar{y}, \bar{z})+\widehat{\operatorname{lip}}_{y} f(\bar{x}, \bar{y})\right], \\
& \operatorname{lip} Q(\bar{y}, \bar{z}) \leq\left\|\nabla_{x} f(\bar{x}, \bar{y})\right\| \cdot \operatorname{reg} S(\bar{x}, \bar{y})+\widehat{\operatorname{lip}}_{y} f(\bar{x}, \bar{y}) .
\end{aligned}
$$


(ii) The solution map $S$ is subregular at $(\bar{x}, \bar{y})$ if and only if $Q$ is calm at $(\bar{y}, \bar{z})$ with the upper estimates of the corresponding exact bounds:

$$
\begin{aligned}
& \text { subreg } S(\bar{x}, \bar{y}) \leq\left\|\left(\nabla_{x} f(\bar{x}, \bar{y})^{*}\right)^{-1}\right\| \cdot\left[\operatorname{clm} Q(\bar{y}, \bar{z})+\widehat{\operatorname{lip}}_{y} f(\bar{x}, \bar{y})\right] \\
& \operatorname{clm} Q(\bar{y}, \bar{z}) \leq\left\|\nabla_{x} f(\bar{x}, \bar{y})\right\| \cdot \operatorname{subreg} S(\bar{x}, \bar{y})+\widehat{\operatorname{lip}}_{y} f(\bar{x}, \bar{y})
\end{aligned}
$$

Proof. It is easy to see from definition (3.19) of strict partial differentiability of the base $f$ at $(\bar{x}, \bar{y})$ that assumption (3.11) of Theorem 3.3 is satisfied with the surjective linear operator $A=\nabla_{x} f(\bar{x}, \bar{y})$ and with the choice of $\mu>0$ such that $\mu \cdot \operatorname{reg} \nabla_{x} f(\bar{x}, \bar{y})$ is arbitrary close to zero. Then all the results of this corollary follow from the corresponding assertions of Theorem 3.3 due to the formula

$$
\operatorname{reg} \nabla_{x} f(\bar{x}, \bar{y})=\left\|\left(\nabla_{x} f(\bar{x}, \bar{y})^{*}\right)^{-1}\right\|
$$

for computing the exact regularity bound of $\nabla_{x} f(\bar{x}, \bar{y})$ given in Proposition 2.5.

Remark 3.6. (Comments on the equivalence and exact bound formulas under strict differentiability.) Observe the following:

(i) In the case of Asplund spaces $X, Y$, and $Z$ and a strictly differentiable mapping $f$ in both variables $(x, y)$ with the surjective partial derivative $\nabla_{x} f(\bar{x}, \bar{y})$, the equivalence in Corollary 3.5(i) is first formulated in [18, Theorem 5.1] with the full proof given in [6, Theorem 5.6] on the basis of coderivative analysis and complete coderivative characterizations of the metric regularity and Lipschitz-like properties of set-valued mapping between Asplund spaces; see [17, Chapter 4]. The proof of Corollary 3.5 (including those of Theorem 3.3 and Lemma 3.1) is based on quite different ideas involving the iterative process of the Lyusternik-Graves type in general Banach spaces, which has been well recognized as a conventional tool of dealing with various problems involving surjective operators; see the above discussions and references. The latter allows us furthermore to establish the equivalence between one-point subregularity and calmness properties in assertion (ii) of Corollary 3.5, which cannot be done via the coderivative analysis of $[6,17,18]$, and to derive in addition tight modulus relationships in (3.20) and (3.21).

(ii) Recall that the inner norm of a positively homogeneous mapping $F: X \rightrightarrows Y$ is defined by

$$
\|F\|^{-}:=\sup _{x \in \mathbb{B}} d(0, F(x))
$$

It is not hard to verify that, for a surjective linear bounded operator $F=A: X \rightarrow Y$, we have

$$
\left\|A^{-1}\right\|^{-}=\left\|\left(A^{*}\right)^{-1}\right\| .
$$

This allows us to conclude, whenever $\widehat{\operatorname{lip}}_{y} f(\bar{x}, \bar{y})=0$, that the estimates in (3.20) and (3.21) reduce, respectively, to the equalities

$$
\operatorname{reg} S(\bar{x}, \bar{y})=\left\|\nabla_{x} f(\bar{x}, \bar{y})\right\|^{-1} \cdot \operatorname{lip} Q(\bar{y}, \bar{z}) \quad \text { and } \quad \operatorname{subreg} S(\bar{x}, \bar{y})=\left\|\nabla_{x} f(\bar{x}, \bar{y})\right\|^{-1} \cdot \operatorname{clm} Q(\bar{y}, \bar{z})
$$

provided that the relative condition number

$$
C\left(\nabla_{x} f(\bar{x}, \bar{y})\right):=\left\|\nabla_{x} f(\bar{x}, \bar{y})\right\| \cdot\left\|\nabla_{x} f(\bar{x}, \bar{y})^{-1}\right\|^{-}
$$

of the operator $\nabla_{x} f(\bar{x}, \bar{y})$ is equal to 1 . We refer the reader to [19] for more information on condition numbers and their applications to numerical aspects of optimization. 
(iii) The equivalence results of Theorem 3.3 and Corollary 3.5 are generally not valid if the field mapping $Q$ in (1.1) depends on the parameter variable $x$. As a simple example, consider a modification of (1.1) with $f(x, y):=x+y$ and $Q(x, y):=-x+y$. All the assumptions of Corollary 3.5 are satisfied, and $Q$ is Lipschitz-like around the origin. At the same time we have $S(x)=\{0\}$ for all $x \in \mathbb{R}$, and thus this solution map is not metrically regular around the origin.

The next result of its independent interest would help us to understand the observation made above in Remark 3.6(iii). It extends to the case of general normed spaces (in fact, the given proof holds in the metric space setting) the one obtained in [17, Theorem 4.16] in Asplund spaces, where on the other hand both mappings are considered to be set-valued.

Proposition 3.7. (Lipschitz-like property and exact bounds under summation.) Let $F: X \rightrightarrows Y$ be a set-valued mapping between normed spaces that is Lipschitz-like around some point $(\bar{x}, \bar{y}) \in \operatorname{gph} F$, and let $g: X \rightarrow Y$ be a single-valued mapping locally Lipschitzian around $\bar{x}$. Then the sum $F+g$ is Lipschitz-like around $(\bar{x} ; \bar{y}+g(\bar{x}))$ with the exact bound estimate

$$
\operatorname{lip}(F+g)(\bar{x}, \bar{y}+g(\bar{x})) \leq \operatorname{lip} F(\bar{x}, \bar{y})+\operatorname{lip} g(\bar{x}) .
$$

Proof. Take arbitrary numbers $\kappa>\operatorname{lip} F(\bar{x}, \bar{y})$ and $\lambda>\operatorname{lip} g(\bar{x})$ and find a constant $\alpha>0$ such that

$$
\begin{gathered}
F(x) \cap \mathbb{B}_{\alpha}(\bar{y}) \subset F\left(x^{\prime}\right)+\kappa\left\|x-x^{\prime}\right\| \mathbb{B} \text { for all } x, x^{\prime} \in \mathbb{B}_{\alpha}(\bar{x}), \\
\left\|g(x)-g\left(x^{\prime}\right)\right\| \leq \lambda\left\|x-x^{\prime}\right\| \text { for all } x, x^{\prime} \in \mathbb{B}_{\alpha}(\bar{x}) .
\end{gathered}
$$

Letting $\beta:=\alpha /(1+\lambda)$, pick $x, x^{\prime} \in \mathbb{B}_{\beta}(\bar{x})$ and $y \in(g+F)(x) \cap \mathbb{B}_{\beta}(\bar{y}+g(\bar{x}))$; there is nothing to prove if this latter intersection is empty. Since

$$
\|y-g(x)-\bar{y}\| \leq\|y-g(\bar{x})-\bar{y}\|+\|g(x)-g(\bar{x})\| \leq \beta+\lambda \beta=\alpha,
$$

there is $\widehat{y} \in F\left(x^{\prime}\right)$ such that $\|\widehat{y}-y+g(x)\| \leq \kappa\left\|x-x^{\prime}\right\|$. Denote further $y^{\prime}:=\widehat{y}+g\left(x^{\prime}\right) \in(g+F)\left(x^{\prime}\right)$ and observe that

$$
\left\|y^{\prime}-y\right\| \leq\|\widehat{y}+g(x)-y\|+\left\|g(x)-g\left(x^{\prime}\right)\right\| \leq(\kappa+\lambda)\left\|x-x^{\prime}\right\| .
$$

The latter justifies the Lipschitz-like property of $g+F$ around $(\bar{x}, \bar{y}+g(\bar{x}))$ with constant $\kappa+\lambda$. Moreover, we arrive at the exact bound estimate (3.23) due to the choice of $\kappa$ and $\lambda$.

If we now suppose that a counterpart of Theorem 3.3 or Corollary 3.5 holds for parameterdependent field mappings $Q=Q(x, y)$, then we could apply it to the solution maps

$$
\widetilde{S}(x):=\{y \in Y \mid 0 \in f(x, y)+\widetilde{Q}(x, y)\}
$$

of such generalized equations with base mappings $f: X \times Y \rightarrow Z$ satisfying the assumptions of the aforementioned results. Given $Q: Y \Rightarrow Z$, form $\widetilde{Q}: X \times Y \Rightarrow Z$ in (3.24) as

$$
\widetilde{Q}(x, y):=-f(x, y)+Q(y)
$$

and observe by Proposition 3.7 that $\widetilde{Q}$ is Lipschitz-like simultaneously with $Q$ around the corresponding points. Since the solution map $\widetilde{S}(x) \equiv\{y \in Y \mid 0 \in Q(y)\}$ to (3.24) with the field $\widetilde{Q}(x, y)$ formed in this way is independent of $x$, it might not be metrically regular while $\widetilde{Q}$ has the Lipschitzlike property; see Remark 3.4(i) for more details. This explains the impossibility of extending the results derived above to generalized equations with parameter-dependent fields.

The last result of this section establishes a counterpart of Theorem 3.3 with the replacement of the base mapping $f$ in (1.1) by its strict estimator around the point in question. This result is generally independent of both Theorem 3.3 and its Corollary 3.5 . 
Theorem 3.8. (Metric regularity and subregularity of solution maps via strict base estimators.) Suppose under the assumptions of Theorem 3.3 that $h: Y \rightarrow Z$ is a strict estimator of the base mapping $f$ in (1.1) with respect to $y$ uniformly in $x$ at $(\bar{x}, \bar{y})$ with constant $\lambda \geq 0$. Then the following assertions hold:

(i) The solution map $S$ in (1.2) is metrically regular around $(\bar{x}, \bar{y})$ if and only if the perturbed field $h+Q$ is Lipschitz-like around $(\bar{y}, 0)$. Furthermore, we has the exact bound estimates:

$$
\begin{aligned}
& \operatorname{reg} S(\bar{x}, \bar{y}) \leq \frac{\operatorname{reg} A \cdot\left[\operatorname{lip}(h+Q)(\bar{y}, 0)+\lambda+\widehat{\operatorname{lip}}_{y} f(\bar{x}, \bar{y})\right]}{1-\mu \cdot \operatorname{reg} A}, \\
& \operatorname{lip}(h+Q)(\bar{y}, 0) \leq \widehat{\operatorname{lip}}_{x} f(\bar{x}, \bar{y}) \cdot \operatorname{reg} S(\bar{x}, \bar{y})+\lambda
\end{aligned}
$$

(ii) The solution map $S$ is metrically subregular at $(\bar{x}, \bar{y})$ if and only if the perturbed field $h+Q$ is calm at $(\bar{y}, 0)$. We have furthermore that

$$
\begin{aligned}
& \text { subreg } S(\bar{x}, \bar{y}) \leq \frac{\operatorname{reg} A \cdot\left[\operatorname{clm}(h+Q)(\bar{y}, 0)+\lambda+\widehat{\operatorname{lip}}_{y} f(\bar{x}, \bar{y})\right]}{1-\mu \cdot \operatorname{reg} A}, \\
& \operatorname{clm}(h+Q)(\bar{y}, 0) \leq \widehat{\operatorname{lip}}_{x} f(\bar{x}, \bar{y}) \cdot \operatorname{subreg} S(\bar{x}, \bar{y})+\lambda
\end{aligned}
$$

Proof. We follow the lines in the proof of Theorem 3.3 with the replacement of $z$ and $z^{\prime}$ therein by $z-h(y)$ and $z^{\prime}-h\left(y^{\prime}\right)$ and with the use of the underlying properties of strict estimators.

\section{Failure of Metric Regularity and Weak Metric Regularity for Major Classes of Parametric Variational Systems}

In this section we implement some equivalence results obtained in Section 3 and the scheme developed in [18] to show that the property of metric regularity around the point in question fails for major variational systems in arbitrary Banach spaces represented as solution maps to rather broad classes of parametric generalized equations (1.1) including, in particular, classical variational inequalities, complementarity problems, KKT systems in mathematical programming, etc. Moreover, we show that the same phenomenon holds not only for the underlying metric regularity property but also for its weak counterpart introduced in this paper. Observe, however, that this does not hold for the at-point metric subregularity property as shown below.

We start with introducing the weak metric regularity concept studied in what follows.

Definition 4.1. (Weak metric regularity.) A set-valued mapping $F: X \rightrightarrows Y$ is WEAKLY METRICALLY REGULAR around $(\bar{x}, \bar{y}) \in \mathrm{gph} F$ if for every neighborhood of $(\bar{x}, \bar{y})$ there exists some point on it such that $F$ is metrically regular around this point.

It is obvious that any mapping $F$ metrically regular around the reference point is always weakly metrically regular around it, but the opposite implication does not hold. Indeed, the latter property can be much weaker than the former one. As a simple example, consider a smooth real function $f: \mathbb{R} \rightarrow \mathbb{R}$ given by $f(x):=x^{2}$ for $x \in \mathbb{R}$. It is easy to see that this function is not metrically regular around $(0,0)$ while it is metrically regular at every point different from the reference one, i.e., it is weakly metrically regular around the origin.

The next theorem on the failure of metric regularity and weak metric regularity under the corresponding pretty mild assumptions is based on the equivalence result of Corollary 3.5(i) and the 
remarkable fact that set-valued monotone mappings and the like with appropriate lower semicontinuity properties turn out to be singe-valued around the reference points. The original result in this direction goes back to Kenderov [11], and then this phenomenon has been well recognized and used in variational analysis; see, e.g., $[1,3,5,13,18,23]$ with the references therein for various manifestations, developments, and applications. We confine ourselves to the implementation of the equivalence results from Corollary 3.5(i) in the conclusions below while similar conclusions can be made on the basis of the more general equivalence results from Theorem 3.3(i) and Theorem 3.8(i).

Theorem 4.2. (Failure of metric regularity and weak metric regularity for solution maps to parametric generalized equations with monotone fields.) Let $f: X \times Y \rightarrow Y^{*}$ be a mapping between Banach spaces that is Lipschitz continuous in a neighborhood of $(\bar{x}, \bar{y}) \in X \times Y$ and strictly partially differentiable at this point with respect to $x$ uniformly in $y$ with the surjective partial derivative $\nabla_{x} f(\bar{x}, \bar{y})$. Assume that the set-valued field mapping $Q: Y \rightrightarrows Y^{*}$ is locally monotone around $\left(\bar{y}, \bar{y}^{*}\right)$ with $\bar{y}^{*}:=-f(\bar{x}, \bar{y}) \in Q(\bar{y})$. The following assertions holds:

(i) If there is no neighborhood of $\bar{y}$ on which $Q$ is entirely single-valued, then the solution map $S$ in (1.2) is not metrically regular around $(\bar{x}, \bar{y})$.

(ii) If there exists a neighborhood of $\bar{y}$ such that for every point $y$ on it there is no neighborhood of $y$ on which $Q$ is entirely single-valued, then $S$ is not weakly metrically regular around $(\bar{x}, \bar{y})$.

Proof. It follows the lines in the proof of [18, Theorem 5.1], where the only assertion (i) was considered in the case of Asplund spaces $X, Y, Y^{*}$ assuming in addition that $f$ is strictly differentiable at $(\bar{x}, \bar{y})$ with respect to both variables $(x, y)$ and that $Q$ is locally closed-graph. The difference between the current setting and the one in the proof of [18, Theorem 5.1] is that we employ now the new equivalence result of Corollary 3.5(i) instead of [6, Theorem 5.6] used in [18].

Remark 4.3. (Calmness plus monotonicity do not imply single-valuedness.) It turns out that, in contrast to robust semicontinuity/Lipschitzian properties, the aforementioned Kenderovtype results that monotonicity implies single-valuedness does not hold for the at-point calmness property. A simple example is given by a set-valued mapping $Q: \mathbb{R} \Rightarrow \mathbb{R}$ defined as

$$
Q(y):= \begin{cases}\emptyset & \text { for } y<0 \\ (-\infty, 0] & \text { for } y=0 \\ \{0\}, & \text { for } y>0\end{cases}
$$

which is is both monotone on $\mathbb{R}$ and calm at $(0,0)$ while not single-valued at the origin. Therefore, a parallel result to Theorem 4.2 would not be valid for metric subregularity.

A striking realization of the non-metric-regularity results of Theorem 4.2 is provided by solution maps for broad and highly important classes of parametric variational inequalities described in the generalized equation form (1.1) with subdifferential field mappings of the type

$$
0 \in f(x, y)+\partial \varphi(y) \text { for } x \in X \text { and } y \in Y \text {, }
$$

where the generating potential $\varphi: X \rightarrow \overline{\mathbb{R}}$ is a convex extended-real-valued function. Note that model (4.1) encompasses, in particular, classical variational inequalities and complementarity problems corresponding to the case of $\varphi(y)=\delta(y ; \Omega)$, the indicator function of a convex set $\Omega$, in the generalized equation formalism (4.1).

The first assertion in the following corollary extends the result of [18, Corollary 5.2], where the base mapping $f$ acts between Asplund spaces and is assumed to be strictly differentiable at $(\bar{x}, \bar{y})$, and where $\psi$ is assumed in addition to be closed/lower semicontinuous around $\bar{y}$. 
Corollary 4.4. (Failure of metric regularity and weak metric regularity for solution maps to parametric variational inequalities.) Let $S: X \rightrightarrows Y$ be the solution map to the parametric variational inequality (4.1) generated by a convex function $\varphi: X \rightarrow \overline{\mathbb{R}}$. Assume that the base mapping $f: X \times Y \rightarrow Y^{*}$ between Banach spaces is Lipschitz continuous around $(\bar{x}, \bar{y})$ with $-f(\bar{x}, \bar{y}) \in \partial \varphi(\bar{y})$ and strictly partially differentiable at this point with respect to $x$ uniformly in $y$ with the surjective partial derivative $\nabla_{x} f(\widetilde{x}, \bar{y})$. Then the following assertions hold:

(i) If the potential $\varphi$ is not Gâteaux differentiable at $\bar{y}$, then the solution map $S$ is not metrically regular around $(\bar{x}, \bar{y})$.

(ii) If the potential $\varphi$ is not Gâteaux differentiable around $\bar{y}$, then the solution map $S$ is not weakly metrically regular around $(\bar{x}, \bar{y})$.

Proof. Observe directly from the definitions of the monotonicity and the subdifferential of convex functions that the subdifferential mapping $Q(y)=\partial \varphi(y)$ is monotone. Thus both conclusions of the corollary follow from the corresponding assertions of Theorem 4.2 and the well-known fact of convex analysis that the subdifferential of $\varphi$ is a singleton at some point if and only if the function is Gâteaux differentiable at this point.

Remark 4.5. (Discussions on the failure of metric regularity.) The following comments illuminate the phenomenon of the failure of metric regularity for solution maps to parametric generalized equations revealed in [18] and in the results given above.

(i) It has been pointed out to us by Terry Rockafellar (personal communication) that the observed phenomenon is in agreement with the generic ill-posedness of inverse problems, which has been recognized and employed in various branches of applied mathematics. Indeed, let us associate with the solution map $S(x)$ in (1.2) the parameter map $P: Y \Rightarrow X$ defined by

$$
P(y):=\{x \in X \mid 0 \in f(x, y)+Q(y)\} .
$$

The parameter map (4.2) has direct motivation from the angle of parameter identification relating to models in which solutions are known from, e.g., experimental data while the generated parameters $x$ are not actually known and should be identified and eventually computed. Observing that $P$ is in fact the inverse mapping to $S$, we deduce from Proposition 2.4(i) that metric regularity of $S$ is equivalent to the Lipschitz-like property of $P$ around the corresponding points. Thus the results of Theorem 4.2 and Corollary 4.4 above as well as those in [18] can be interpreted as revealing an inherent instability/ill-posedness of the parameter identification problem (4.2). In this way we conclude that the obtained results on the failure of metric regularity for variational systems described by (1.2) can be treated as yet another manifestation of ill-posedness of inverse problems.

(ii) In the recent paper [22], Robinson discussed the failure of metric regularity observed in [18] and some related "bunching phenomena" in several areas of optimization and variational analysis. He developed a powerful reparametrization approach to the class of nonsmooth equations considered in [22], which dealt with both primal and dual variables and allowed him, in particular, to avoid the aforementioned unpleasant phenomena; we refer the reader to [22] for more details.

(iii) Aussel et al. [3] extended the non-metric-regularity result of [18, Theorem 5.1] to the class of quasivariational inequalities generated by quasimonotone set-valued field mappings $Q$ in the framework of (1.1). The proof of this result in [3, Theorem 5.1] is based on the new singledirectional property of such operators established therein under the Lipschitz-like assumption in Banach spaces and the aforementioned equivalence result of [6, Theorem 5.6] in the Asplund space setting. Thus the result of [3, Theorem 5.1] can be now extended to general Banach spaces by using the single-directional property of quasimonotone operators from [3] and the new equivalence relationships from Theorem 3.3 and Corollary 3.5 obtained above. 
In the final theorem of this section on the failure of metric regularity and its weak counterpart, we aim to extend the results of Corollary 4.4 to the case of parameterized generalized equations with the so-called composite fields $Q(y)=(\partial \varphi \circ g)(y)$. To proceed, derive first the following proposition of its own interest on preserving the Lipschitz-like property under compositions of mappings between Banach spaces establishing also tight quantitative relationships between the exact bounds of the corresponding Lipschitzian moduli. Assertion (i) of this proposition extends that of [17, Corollary 4.15] from Asplund to Banach spaces (note that the proof given below holds in fact for general metric spaces), while assertion (ii) seems to be new even in finite dimensions.

Proposition 4.6. (Preservation of the Lipschitz-like property under compositions.) Let $g: X \rightarrow Y$ and $F: Y \rightrightarrows Z$ be mappings between Banach spaces, and let $\bar{z} \in(F \circ g)(\bar{x})$. Assume that $g$ is Lipschitz continuous around $\bar{x}$. Then the following assertions hold:

(i) The composition $F \circ g$ has the Lipschitz-like property around $(\bar{x}, \bar{z})$ provided that $F$ enjoys this property around $(g(\bar{x}), \bar{z})$. In this case

$$
\operatorname{lip}(F \circ g)(\bar{x}, \bar{z}) \leq \operatorname{lip} g(\bar{x}) \cdot \operatorname{lip} F(g(\bar{x}), \bar{z}) .
$$

(ii) If conversely $F \circ g$ is Lipschitz-like around $(\bar{x}, \bar{z})$ and if $g$ is strictly differentiable at $\bar{x}$ with the surjective derivative $\nabla g(\bar{x})$, then $F$ is Lipschitz-like around $(g(x), \bar{z})$ with

$$
\operatorname{lip} F(g(\bar{x}), \bar{z}) \leq\left\|\left(\nabla g(\bar{x})^{*}\right)^{-1}\right\| \cdot \operatorname{lip}(F \circ g)(\bar{x}, \bar{z}) .
$$

Proof. To justify (i), take arbitrary constants $\kappa>\operatorname{lip} F(g(\bar{x}), \bar{z})$ and $\lambda>\operatorname{lip} g(\bar{x})$ and then find a positive number $a$ such that

$$
\begin{gathered}
F(y) \cap \mathbb{B}_{a}(\bar{z}) \subset F\left(y^{\prime}\right)+\kappa\left\|y-y^{\prime}\right\| \mathbb{B} \text { for all } y, y^{\prime} \in \mathbb{B}_{a}(g(\bar{x})) \text { and } \\
\|g(x)-g(\bar{x})\| \leq \lambda\left\|x-x^{\prime}\right\| \text { for all } x, x^{\prime} \in \mathbb{B}_{a}(\bar{x})
\end{gathered}
$$

Take further $b \in(0, a]$ satisfying $\lambda b \leq a$ and pick any $x, x^{\prime} \in \mathbb{B}_{b}(\bar{x})$ and $z \in(F \circ g)(x) \cap \mathbb{B}_{b}(\bar{z})$. Then we have the inequalities

$$
\|g(u)-g(\bar{x})\| \leq \lambda\|u-\bar{x}\| \leq \lambda b \leq a \text { for } u=x, x^{\prime} .
$$

From the Lipschitz-like property of $F$ around $(g(\bar{x}), \bar{z})$ and the relationships above, observe that

$$
z \in F(g(x)) \cap \mathbb{B}_{a}(\bar{z}) \subset F\left(g\left(x^{\prime}\right)\right)+\kappa\left\|g(x)-g\left(x^{\prime}\right)\right\| \mathbb{B},
$$

i.e., there exists $z^{\prime} \in(F \circ g)\left(x^{\prime}\right)$ satisfying the estimates

$$
\left\|z^{\prime}-z\right\| \leq \kappa\left\|g(x)-g\left(x^{\prime}\right)\right\| \leq \kappa \lambda\left\|x-x^{\prime}\right\| .
$$

Since $\kappa$ and $\lambda$ were chosen arbitrarily close to $\operatorname{lip} F(g(\bar{x}), \bar{z})$ and lip $g(\bar{x})$, we arrive the Lipschitz-like property of the composition $F \circ g$ around $(\bar{x}, \bar{z})$ with the exact bound estimate (4.3).

To proceed with assertion (ii), take arbitrary $\kappa>\operatorname{lip} F(g(\bar{x}), \bar{z})$ and $\lambda>\operatorname{reg} \nabla g(\bar{x})=\left\|\left(\nabla g(\bar{x})^{*}\right)^{-1}\right\|$, where the latter equality holds due to Proposition 2.5. Define a mapping $\Gamma: Y \rightrightarrows X$ by

$$
\Gamma(y):=\{x \in X \mid g(x)=y\}, \quad y \in Y .
$$


Employing then Lemma 3.1, where the constant $\mu>0$ can be chosen arbitrarily small due to the differentiability of $g$ in (4.5), find a number $\alpha>0$ such that

$$
\Gamma\left(y^{\prime}\right) \cap \mathbb{B}_{\alpha}(\bar{x}) \subset \Gamma(y)+\lambda\left\|y-y^{\prime}\right\| \mathbb{B} \text { for all } y, y^{\prime} \in \mathbb{B}_{\alpha}(g(\bar{x})) .
$$

By the assumed Lipschitz-like property of $F \circ g$ around $(\bar{x}, \bar{z})$ there is $a \in(0, \alpha)$ for which

$$
(F \circ g)(x) \cap \mathbb{B}_{a}(\bar{z}) \subset(F \circ g)\left(x^{\prime}\right)+\kappa\left\|x-x^{\prime}\right\| \mathbb{B} \text { whenever } x, x^{\prime} \in \mathbb{B}_{a}(\breve{x}) \text {. }
$$

Take further $b \in(0, a]$ satisfying $3 \lambda b \leq a$ and then pick $y, y^{\prime} \in \mathbb{B}_{b}(g(\bar{x}))$ and $z \in F(y) \cap \mathbb{B}_{b}(\bar{z})$; we are done if there is no such $z$. It follows from (4.6) and the definition of $\Gamma$ that

$$
\bar{x} \in \Gamma(g(\bar{x})) \cap \mathbb{B}_{\alpha}(\bar{x}) \subset \Gamma(y)+\lambda\|y-g(\bar{x})\| \mathbb{B},
$$

which ensures the existence of $x \in \Gamma(y)$ with

$$
\|x-\bar{x}\| \leq \lambda\|y-g(\bar{x})\| \leq \lambda b \leq a \leq \alpha .
$$

Employing (4.6) again, we get the inclusion

$$
x \in \Gamma(y) \cap \mathbb{B}_{\alpha}(\bar{x}) \subset \Gamma\left(y^{\prime}\right)+\lambda\left\|y-y^{\prime}\right\| \mathbb{B},
$$

which implies in turn the existence of $x^{\prime} \in \Gamma\left(y^{\prime}\right)$ satisfying $\left\|x-x^{\prime}\right\| \leq \lambda\left\|y-y^{\prime}\right\|$. Thus

$$
\left\|x^{\prime}-\bar{x}\right\| \leq\left\|x-x^{\prime}\right\|+\|x-\bar{x}\| \leq 3 \lambda b \leq a .
$$

The latter gives, by the assumed Lipschitz-like property (4.7), that

$$
\begin{gathered}
z \quad \in F(y) \cap \mathbb{B}_{b}(\bar{z})=F(g(x)) \cap \mathbb{B}_{b}(\bar{z}) \subset(F \circ g)(x) \cap \mathbb{B}_{a}(\bar{z}) \\
\subset(F \circ g)\left(x^{\prime}\right)+\kappa\left\|x-x^{\prime}\right\| \mathbb{B}=F\left(y^{\prime}\right)+\kappa\left\|x-x^{\prime}\right\| \mathbb{B},
\end{gathered}
$$

and hence there is $z^{\prime} \in F\left(y^{\prime}\right)$ satisfying $\left\|z-z^{\prime}\right\| \leq \kappa\left\|x-x^{\prime}\right\| \leq \kappa \lambda\left\|y-y^{\prime}\right\|$. By the choice of $\kappa$ and $\lambda$ above we thus arrive at the Lipschitz-like property of $F$ around $(\bar{x}, \bar{z})$ with the exact bound estimate (4.4). This completes the proof of the proposition.

Now we are ready to derive the aforementioned results on the failure of the metric regularity and weak metric regularity properties for solution maps to the generalized equations (1.1) with composite fields. The first statement of the following theorem extends the result of [18, Theorem 5.4] obtained therein in the Asplund space setting under additional assumptions on the strict differentiability of the base mapping $f$ with respect to both variables at $(\bar{x}, \bar{y})$ and the lower semicontinuity of the potential $\varphi$ around the point $g(\bar{y})$ in the notation below.

Theorem 4.7. (Failure of metric regularity and weak metric regularity for solution maps to parametric generalized equations with composite fields.) Let $S: X \rightrightarrows Y$ be the solution map to the generalized equation (1.1) with the composite field $Q(y)=(\partial \varphi \circ g)(y)$, where $f: X \times Y \rightarrow W^{*}$ is a mapping between Banach spaces that is Lipschitz continuous around $(\bar{x}, \bar{y}) \in \operatorname{gph} S$ and strictly partially differentiable at this point with respect to $x$ uniformly in $y$ with the surjective partial derivative $\nabla_{x} f(\bar{x}, \bar{y})$, where $g: Y \rightarrow W$ is strictly differentiable at $\bar{y}$ with the surjective derivative $\nabla g(\bar{y})$, and where $\varphi: W \rightarrow \overline{\mathbb{R}}$ is a convex function finite at $\bar{w}:=g(\bar{y})$. Then the following assertions are satisfied:

(i) If the potential $\varphi$ is not Gâteaux differentiable at $\bar{w}$, then the solution map $S$ is not metrically regular around $(\bar{x}, \bar{y})$.

(ii) If the potential $\varphi$ is not Gâteaux differentiable around $\bar{w}$, then the solution map $S$ is not weakly metrically regular around $(\bar{x}, \bar{y})$. 
Proof. It is sufficient to justify assertion (i) observing that the proof of assertion (ii) is similar by taking into account Definition 4.1 of weak metric regularity. Arguing by contradiction, suppose that the solution map $S: X \rightrightarrows Y$ given by

$$
S(x):=\{y \in Y \mid 0 \in f(x, y)+(\partial \varphi \circ g)(y)\}
$$

is metrically regular around $(\bar{x}, \bar{y})$ under the assumptions made. Then, by Corollary $3.5(\mathrm{i})$, the mapping $Q(y)=(\partial \varphi \circ g)(y)$ is Lipschitz-like around $\left(\bar{y}, \bar{w}^{*}\right) \in \operatorname{gph}(\partial \varphi \circ g)$, where $\bar{w}^{*}:=-f(\bar{x}, \bar{y})$. Since $g$ has the surjective derivative $\nabla g(\bar{y})$, we conclude from Proposition 4.6(ii) that the subdifferential mapping $\partial \varphi: W \rightrightarrows W^{*}$ is Lipschitz-like around $\left(\bar{w}, \bar{w}^{*}\right)$. The latter contradicts the assumption that $\varphi$ is not Gâteaux differentiable at $\bar{w}$; cf. the proofs of Corollary 4.4 and Theorem 4.2.

\section{Lipschitzian Properties of Solution Maps via Metric Regularity of Fields in Generalized Equations}

The main theme of this section is to obtain results, which are reverse to some of those derived in Section 3. Namely, we aim to establish the equivalence between the Lipschitz-like property of solution maps to generalized equations and the metric regularity of field mappings around the corresponding points, with tight quantitative relationships for the associated exact bounds of moduli. It is worth emphasizing that the results obtained in this section are fully independent of those from Section 3 due to the asymmetry between solution maps and fields in generalized equations. Moreover, the equivalence between the at-point properties of calmness for fields and metric subregularity for soIution maps established in Section 3 does not generally hold in the reverse framework considered in this section. Nevertheless, we reveal some settings of such an "at-point" equivalence and explore the results obtained in this way for deriving new verifiable conditions for metric subregularity and calmness of solution maps to generalized equations.

Let us start with a reverse counterpart of Theorem 3.3(i). It is worth mentioning that, in contrast to the results of Section 3, the converse assertions in the next theorem and its further counterparts established below do not require the surjectivity assumption on the approximating/partial derivative operators under consideration.

Theorem 5.1. (Lipschitz-like property of solution maps via metric regularity of fields in generalized equations.) Let $f: X \times Y \rightarrow Z$ be a mapping between Banach spaces that is Lipschitz continuous on a neighborhood $U \times V$ of $(\bar{x}, \bar{y}) \in X \times Y$, and let $Q: Y \rightrightarrows Z$ be a set-valued field mapping with $\bar{z}:=-f(\bar{x}, \bar{y}) \in Q(\bar{y})$ such that the graph of $Q$ is locally closed around $(\bar{y}, \bar{z})$. The following assertions hold:

(i) Assume that $A \in \mathcal{L}(X, Z)$ is a surjective linear operator satisfying (3.1) with some $\mu \geq 0$. If the solution map $S: X \rightrightarrows Y$ in (1.2) is Lipschitz-like around $(\bar{x}, \bar{y})$ and if the condition

$$
\operatorname{reg} A \cdot\left[\mu+\operatorname{lip} S(\bar{x}, \bar{y}) \cdot \widehat{\operatorname{lip}}_{y} f(\breve{x}, \bar{y})\right]<1
$$

is fulfilled, then $Q$ is metrically regular around $(\bar{y}, \bar{z})$ with the exact bound estimate

$$
\operatorname{reg} Q(\bar{y}, \bar{z}) \leq \frac{\operatorname{lip} S(\bar{x}, \bar{y}) \cdot \operatorname{reg} A}{1-\operatorname{reg} A \cdot\left[\mu+\operatorname{lip} S(\bar{x}, \bar{y}) \cdot \widehat{\operatorname{lip}}_{y} f(\bar{x}, \bar{y})\right]} .
$$

(ii) Conversely, assume that $Q$ is metrically regular around $(\bar{y}, \bar{z})$ and that the condition

$$
\widehat{\operatorname{lip}}_{y} f(\bar{x}, \bar{y}) \cdot \operatorname{reg} Q(\bar{y}, \bar{z})<1
$$


is satisfied. Then $S$ is Lipschitz-like around $(\bar{x}, \bar{y})$ with the exact bound estimate

$$
\operatorname{lip} S(\bar{x}, \bar{y}) \leq \frac{\operatorname{reg} Q(\bar{y}, \bar{z}) \cdot \widehat{\operatorname{lip}}_{x} f(\bar{x}, \bar{y})}{1-\operatorname{reg} Q(\bar{y}, \bar{z}) \cdot \widehat{\operatorname{lip}}_{y} f(\bar{x}, \bar{y})} .
$$

Proof. To justify (i), take $\theta>\operatorname{lip} S(\bar{x}, \bar{y})$ and apply Lemma 3.1. Then for any $\eta>\operatorname{lip} f(\bar{x}, \bar{y})$ and any $\gamma>\operatorname{reg} A$ satisfying $\gamma(\mu+\theta \eta)<1$ we find a constant $\alpha>0$ such that

$$
\Gamma\left(y^{\prime}, z^{\prime}\right) \cap \mathbb{B}_{\alpha}(\bar{x}) \subset \Gamma(y, z)+\frac{\gamma}{1-\gamma \mu}\left(\eta\left\|y-y^{\prime}\right\|+\left\|z-z^{\prime}\right\|\right) \mathbb{B}
$$

for every $(y, z),\left(y^{\prime}, z^{\prime}\right) \in \mathbb{B}_{\alpha}(\bar{y}) \times \mathbb{B}_{\alpha}(\bar{z})$, where $\Gamma(y, z):=\{x \in X \mid f(x, y)+z=0\}$. Make $\alpha$ smaller if necessary so that $\operatorname{gph} Q \cap \mathbb{B}_{\alpha}(\bar{y}) \times \mathbb{B}_{\alpha}(\bar{z})$ is closed and that

$$
S(x) \cap \mathbb{B}_{\alpha}(\bar{x}) \subset S\left(x^{\prime}\right)+\theta\left\|x-x^{\prime}\right\| \mathbb{B} \text { whenever } x, x^{\prime} \in \mathbb{B}_{\alpha}(\bar{x}) .
$$

Select next $\beta \in(0, \alpha)$ satisfying the estimates

$$
\frac{2 \beta \theta \gamma^{2} \eta}{1-\gamma(\mu+\theta \eta)}+\frac{\gamma \beta(\eta+3)}{1-\gamma \mu} \leq \alpha \text { and } \frac{2 \beta \theta \gamma}{1-\gamma(\mu+\theta \eta)}\left(\frac{\eta \theta \gamma}{1-\gamma \mu}+1\right)+\beta \leq \alpha .
$$

Pick $z, z^{\prime} \in \mathbb{B}_{\beta}(\bar{z})$ and $y^{\prime} \in Q^{-1}\left(z^{\prime}\right) \cap \mathbb{B}_{\beta}(\bar{y})$ observing that we are done if such a point $y^{\prime}$ does not exist. We aim to construct by induction two sequences $\left\{x_{k}\right\} \subset \mathbb{B}_{\alpha}(\bar{x})$ and $\left\{y_{k}\right\} \subset \mathbb{B}_{\alpha}(\bar{y})$ such that the pair $\left(x_{k}, y_{k}\right)$ converges to some point $(x, y)$ satisfying $z=-f(x, y), y \in Q^{-1}(z)$, and

$$
\left\|y-y^{\prime}\right\| \leq \frac{\theta \gamma}{1-\gamma(\mu+\theta \eta)}\left\|z-z^{\prime}\right\| .
$$

Start with $y_{0}:=y^{\prime}$ and, by $\bar{x} \in \Gamma(\bar{y}, \bar{z})$ and (5.5), find $x_{0} \in \Gamma\left(y_{0}, z^{\prime}\right)$ such that

$$
\left\|x_{0}-\bar{x}\right\| \leq \frac{\gamma}{1-\gamma \mu}\left(\eta\left\|y_{0}-\bar{y}\right\|+\left\|z^{\prime}-\bar{z}\right\|\right) \leq \frac{\gamma \beta}{1-\gamma \mu}(\eta+1) \leq \alpha
$$

Using again (5.5) but now with $x_{0} \in \Gamma\left(y_{0}, z^{\prime}\right)$ and $\left(y_{0}, z\right)$, find $x_{1} \in \Gamma\left(y_{0}, z\right)$ satisfying the estimate $\left\|x_{1}-x_{0}\right\| \leq \gamma /(1-\gamma \mu)\left\|z-z^{\prime}\right\|$. Then we have

$$
\left\|x_{1}-\bar{x}\right\| \leq\left\|x_{1}-x_{0}\right\|+\left\|x_{0}-\bar{x}\right\| \leq \frac{\gamma \beta}{1-\gamma \mu}(\eta+3) \leq \alpha .
$$

It follows from (5.6) that there is $y_{1} \in S\left(x_{1}\right)$ with $\left\|y_{1}-y_{0}\right\| \leq \theta\left\|x_{1}-x_{0}\right\|$. Thus

$$
\left\|y_{1}-y_{0}\right\| \leq \frac{\theta \gamma}{1-\gamma \mu}\left\|z-z^{\prime}\right\|
$$

which in turn implies the estimate

$$
\left\|y_{1}-\bar{y}\right\| \leq\left\|y_{1}-y_{0}\right\|+\left\|y_{0}-\bar{y}\right\| \leq \frac{2 \beta \theta \gamma}{1-\gamma \mu}+\beta \leq \alpha
$$

Utilizing once more (5.5), find $x_{2} \in \Gamma\left(y_{1}, z\right)$ such that $\left\|x_{2}-x_{1}\right\| \leq \gamma \eta /(1-\gamma \mu)\left\|y_{1}-y_{0}\right\|$. Hence

$$
\left\|x_{2}-\bar{x}\right\| \leq\left\|x_{2}-x_{1}\right\|+\left\|x_{1}-\bar{x}\right\| \leq \frac{\gamma \beta}{1-\gamma \mu}\left(\frac{2 \eta \theta \gamma}{1-\gamma \mu}+\eta+3\right) \leq \alpha
$$


and then, by (5.6), there is $y_{2} \in S\left(x_{2}\right)$ with $\left\|y_{2}-y_{1}\right\| \leq \theta\left\|x_{2}-x_{1}\right\|$. This yields

$$
\left\|y_{2}-\bar{y}\right\| \leq\left\|y_{2}-y_{1}\right\|+\left\|y_{1}-\bar{y}\right\| \leq \frac{2 \beta \theta \gamma}{1-\gamma \mu}\left(\frac{\eta \theta \gamma}{1-\gamma \mu}+1\right)+\beta \leq \alpha .
$$

Now we proceed by induction. Take points $x_{k} \in \mathbb{B}_{\alpha}(\bar{x})$ and $y_{k} \in \mathbb{B}_{\alpha}(\bar{y})$ satisfying the conditions

$$
\begin{aligned}
y_{k} \in S\left(x_{k}\right), \quad\left\|y_{k}-y_{k-1}\right\| & \leq \theta\left\|x_{k}-x_{k-1}\right\|, \\
x_{k} \in \Gamma\left(y_{k-1}, z\right), & \text { and } \quad\left\|x_{k}-x_{k-1}\right\| \leq \frac{\gamma \eta}{1-\gamma \mu}\left\|y_{k-1}-y_{k-2}\right\|
\end{aligned}
$$

for all $k=2, \ldots, n$. Using condition (5.5), find a point $x_{n+1} \in \Gamma\left(y_{n}, z\right)$ for which we have the estimate $\left\|x_{n+1}-x_{n}\right\| \leq \gamma \eta /(1-\gamma \mu)\left\|y_{n}-y_{n-1}\right\|$. The induction assumption (5.7) yields that

$$
\begin{aligned}
\left\|x_{n+1}-\bar{x}\right\| & \leq \sum_{k=2}^{n+1}\left\|x_{k}-x_{k-1}\right\|+\left\|x_{1}-\bar{x}\right\| \leq \sum_{k=2}^{n+1}\left(\frac{\theta \gamma \eta}{1-\gamma \mu}\right)^{n-2}\left\|x_{2}-x_{1}\right\|+\left\|x_{1}-\bar{x}\right\| \\
& \leq \frac{\gamma \eta}{1-\gamma \mu} \sum_{k=2}^{n+1}\left(\frac{\theta \gamma \eta}{1-\gamma \mu}\right)^{k-1}\left\|y_{1}-y_{0}\right\|+\left\|x_{1}-\bar{x}\right\| \\
& \leq \frac{\gamma \eta}{1-\gamma(\mu+\theta \eta)}\left\|y_{1}-y_{0}\right\|+\left\|x_{1}-\bar{x}\right\| \\
& \leq \frac{2 \beta \theta \gamma^{2} \eta}{(1-\gamma \mu)(1-\gamma(\mu+\theta \eta))}+\frac{\gamma \beta(\eta+3)}{1-\gamma \mu} \leq \alpha .
\end{aligned}
$$

Applying (5.6), find $y_{n+1} \in S\left(x_{n+1}\right)$ such that $\left\|y_{n+1}-y_{n}\right\| \leq \theta\left\|x_{n+1}-x_{n}\right\|$. Consequently

$$
\begin{aligned}
\left\|y_{n+1}-\bar{y}\right\| & \leq \sum_{k=1}^{n+1}\left\|y_{k}-y_{k-1}\right\|+\left\|y_{0}-\bar{y}\right\| \leq \sum_{k=1}^{n+1}\left(\frac{\theta \gamma \eta}{1-\gamma \mu}\right)^{k-1}\left\|y_{1}-y_{0}\right\|+\beta \\
& \leq \frac{1-\gamma \mu}{1-\gamma(\mu+\theta \eta)}\left\|y_{1}-y_{0}\right\|+\beta \leq \frac{2 \beta \theta \gamma}{1-\gamma(\mu+\theta \eta)}+\beta \leq \alpha,
\end{aligned}
$$

which completes the induction step. This gives us two Cauchy sequences $\left\{x_{k}\right\}$ and $\left\{y_{k}\right\}$ converging thus to some points $x \in \mathbb{B}_{\alpha}(\bar{x})$ and $y \in \mathbb{B}_{\alpha}(\bar{y})$, respectively. From the third condition in (5.7) and the continuity of $f$, we get $z=-f(x, y)$. Furthermore, the first property in (5.7) and the local closedness of the graph of $Q$ ensure that $0 \in f(x, y)+Q(y)$, i.e., $y \in Q^{-1}(z)$. Finally, the second equation in (5.7) implies that

$$
\begin{aligned}
\left\|y_{n}-y^{\prime}\right\| & \leq \sum_{k=1}^{n}\left\|y_{k}-y_{k-1}\right\| \leq \sum_{k=1}^{n}\left(\frac{\theta \gamma \eta}{1-\gamma \mu}\right)^{k-1}\left\|y_{1}-y_{0}\right\| \\
& \leq \frac{1-\gamma \mu}{1-\gamma(\mu+\theta \eta)}\left\|y_{1}-y_{0}\right\| \leq \frac{\theta \gamma}{1-\gamma(\mu+\theta \eta)}\left\|z-z^{\prime}\right\| .
\end{aligned}
$$

By passing to the limit as $n \rightarrow \infty$ in the latter expression and taking into account the choice of the constants above, we conclude that the field mapping $Q$ is metrically regular around $(\bar{y}, \bar{z})$ with the exact regularity bound satisfying (5.2). This completes the proof of the first part of the theorem.

To justify assertion (ii), suppose that $Q$ is metrically regular around $(\widetilde{y}, \bar{z})$ and, by the assumptions made, select arbitrary constants $\left(\kappa, \eta_{x}, \eta_{y}\right)$ satisfying

$$
\kappa>\operatorname{reg} Q(\bar{y}, \bar{z}), \quad \eta_{x}>\widehat{\operatorname{lip}}_{x} f(\bar{x}, \bar{y}), \text { and } \eta_{y}>\widehat{\operatorname{lip}}_{y} f(\bar{x}, \bar{y})
$$


Due to the metric regularity of $Q$ around $(\bar{y}, \bar{z})$ and the choice of $\kappa$ there is $\alpha>0$ such that

$$
\begin{gathered}
d\left(y, Q^{-1}(z)\right) \leq \kappa d(z, Q(y)) \text { for all }(y, z) \in \mathbb{B}_{\alpha}(\bar{y}) \times \mathbb{B}_{\alpha}(\bar{z}) \text { and } \\
\left\|f(x, y)-f\left(x^{\prime}, y^{\prime}\right)\right\| \leq \eta_{x}\left\|x-x^{\prime}\right\|+\eta_{y}\left\|y-y^{\prime}\right\| \text { whenever }(x, y),\left(x^{\prime}, y^{\prime}\right) \in \mathbb{B}_{\alpha}(\bar{x}) \times \mathbb{B}_{\alpha}(\bar{y}) .
\end{gathered}
$$

Choose further a positive constant $\beta$ such that

$$
\beta+\frac{2 \kappa \eta_{x} \beta}{1-\kappa \eta_{y}}<\alpha \text { and }\left(\eta_{x}+\eta_{y}\right) \beta+\frac{2 \kappa \eta_{x} \eta_{y} \beta}{1-\kappa \eta_{y}}<\alpha
$$

and then take $x, x^{\prime} \in \mathbb{B}_{\beta}(\bar{x})$ with $x \neq x^{\prime}$ and $y \in S(x) \cap \mathbb{B}_{\beta}(\bar{y})$; we are done if there is no such $y \in Y$. Define a set-valued mapping $\Phi: Y \rightrightarrows Y$ by

$$
\Phi(u):=Q^{-1}\left(-f\left(x^{\prime}, u\right)\right) \text { for } u \in Y
$$

and show that $\Phi$ satisfies all the assumptions of Theorem 2.1. To proceed, pick $\varepsilon>0$ and denote $a_{\varepsilon}:=\left(\kappa \eta_{x} /\left(1-\kappa \eta_{y}\right)+\varepsilon\right)\left\|x-x^{\prime}\right\|$. If $u \in \mathbb{B}_{a_{\varepsilon}}(y)$, then

$$
\|u-\bar{y}\| \leq a_{\varepsilon}+\beta \leq \frac{2 \kappa \eta_{x} \beta}{1-\kappa \eta_{y}}+2 \varepsilon \beta+\beta .
$$

Making $\varepsilon$ smaller if necessary, we can always assume that the latter expression is less than $\alpha$; this can be done due to (5.8). Furthermore, we get the estimates

$\left\|-f\left(x^{\prime}, u\right)-\bar{z}\right\| \leq\left\|f\left(x^{\prime}, u\right)-f(\bar{x}, \bar{y})\right\| \leq \eta_{x}\left\|x^{\prime}-\bar{x}\right\|+\eta_{y}\|u-\bar{y}\| \leq \eta_{x} \beta+\frac{2 \kappa \eta_{x} \eta_{y} \beta}{1-\kappa \eta_{y}}+2 \varepsilon \beta \eta_{y}+\beta \eta_{y}$

where the last number can be also made smaller than $\alpha$ by adjusting $\varepsilon$. Thus the mapping $\Phi$ is well defined on the ball $\mathbb{B}_{a_{\varepsilon}}(y)$ satisfying

$$
\begin{aligned}
d(y, \Phi(y)) & =d\left(y, Q^{-1}\left(-f\left(x^{\prime}, y\right)\right)\right) \leq \kappa d\left(-f\left(x^{\prime}, y\right), Q(y)\right) \\
& \leq \kappa\left\|f\left(x^{\prime}, y\right)-f(x, y)\right\| \leq \kappa \eta_{x}\left\|x-x^{\prime}\right\|<a_{\varepsilon}\left(1-\kappa \eta_{y}\right),
\end{aligned}
$$

which verifies assumption (i) of Theorem 2.1. Moreover, for $u, v \in \mathbb{B}_{a_{\varepsilon}}(y)$ we have

$$
\begin{aligned}
e\left(\Phi(u) \cap \mathbb{B}_{a_{\varepsilon}}(y), \Phi(v)\right) & =\sup \left\{d\left(w, Q^{-1}\left(-f\left(x^{\prime}, v\right)\right)\right) \mid w \in \Phi(u) \cap \mathbb{B}_{a_{\varepsilon}}(y)\right\} \\
& \leq \kappa \sup \left\{d\left(-f\left(x^{\prime}, v\right), Q(w)\right) \mid-f\left(x^{\prime}, u\right) \in Q(w), w \in \mathbb{B}_{a_{\varepsilon}}(y)\right\} \\
& \leq \kappa\left\|f\left(x^{\prime}, u\right)-f\left(x^{\prime}, v\right)\right\| \leq \kappa \eta_{y}\|u-v\|,
\end{aligned}
$$

which verifies the assumption (ii) of the aforementioned theorem. Applying this contraction principle, we find a fixed point $y_{\varepsilon} \in \Phi\left(y_{\varepsilon}\right) \cap \mathbb{B}_{a_{\varepsilon}}(y)$. The latter means that

$$
y_{\varepsilon} \in S\left(x^{\prime}\right) \text { and }\left\|y_{\varepsilon}-y\right\| \leq\left(\frac{\kappa \eta_{x}}{1-\kappa \eta_{y}}+\varepsilon\right)\left\|x-x^{\prime}\right\|
$$

Due to the arbitrary choice of the number $\varepsilon>0$ above, the last estimate ensures the Lipschitz-like property of the solution map $S$ around $(\bar{x}, \bar{y})$ with the exact bound formula (5.4) and thus completes the proof of the theorem. 
Note that both estimates (5.2) and (5.4) in Theorem 5.1 hold as equalities for some mappings $f$ and $Q$ in (1.1). Indeed, take $f$ and $Q$ as in Remark 3.4(ii) above. It can be easily checked that $\operatorname{reg} Q=1 /|c|$ and $\operatorname{lip} S=|a| /|b+c|$, which thus give the equality in (5.2) when $|b+c|=|b|+|c|$ and the equality in (5.4) when $|b+c|=|c|-|b|$.

The next result provides consequences of both assertions of Theorem 5.1 in the case when $f$ is strictly partially differentiable with respect to the parameter variable. To the best of our knowledge, the first results establishing relationships between the Lipschitz-like property of solution maps and metric regularity of fields in generalized equations with strictly differentiable bases were obtained in [16, Corollary 5.10] in the finite-dimensional setting under the assumption that $\nabla_{y} f(\bar{x}, \bar{y})=0$. The latter ensures that both conditions (5.3) and (5.9) in the following corollary are satisfied.

Corollary 5.2. (Equivalence between the Lipschitz-like property of solutions maps and metric regularity of fields for generalized equations with strictly differentiable bases.) Let $f: X \times Y \rightarrow Z$ be a mapping between Banach spaces that is locally Lipschitzian around $(\bar{x}, \bar{y})$ and strictly partially differentiable at this point with respect to $x$ uniformly in $y$ with the partial derivative $\nabla_{x} f(\bar{x}, \bar{y})$, and let $Q: Y \Rightarrow Z$ be a set-valued mapping with $\bar{z}:=-f(\bar{x}, \bar{y}) \in Q(\bar{y})$ that is closed-graph around $(\bar{y}, \bar{z})$. The following assertions hold:

(i) Assume that the operator $\nabla_{x} f(\bar{x}, \bar{y}): X \rightarrow Z$ is surjective, that the solution map $S$ in $(1.2)$ is Lipschitz-like around $(\bar{x}, \bar{y})$, and that the condition

$$
\left\|\left(\nabla_{x} f(\bar{x}, \bar{y})^{*}\right)^{-1}\right\| \cdot \operatorname{lip} S(\bar{x}, \bar{y}) \cdot \widehat{\operatorname{lip}}_{y} f(\bar{x}, \bar{y})<1
$$

is satisfied. Then $Q$ is metrically regular around $(\bar{y}, \bar{z})$ with the exact bound estimate

$$
\operatorname{reg} Q(\bar{y}, \bar{z}) \leq \frac{\operatorname{lip} S(\bar{x}, \bar{y}) \cdot\left\|\left(\nabla_{x} f(\bar{x}, \bar{y})^{*}\right)^{-1}\right\|}{1-\left\|\left(\nabla_{x} f(\bar{x}, \bar{y})^{*}\right)^{-1}\right\| \cdot \operatorname{lip} S(\bar{x}, \bar{y}) \cdot \widehat{\operatorname{lip}}_{y} f(\bar{x}, \bar{y})} .
$$

If we suppose in addition that $\widehat{\operatorname{lip}}_{y} f(\bar{x}, \bar{y})=0$, then

$$
\operatorname{reg} Q(\bar{y}, \bar{z}) \leq \operatorname{lip} S(\bar{x}, \bar{y}) \cdot\left\|\left(\nabla_{x} f(\bar{x}, \bar{y})^{*}\right)^{-1}\right\| .
$$

(ii) Conversely, suppose that $Q$ is metrically regular around $(\bar{y}, \bar{z})$ and that condition (5.3) is satisfied. Then $S$ is Lipschitz-like around $(\bar{x}, \bar{y})$ with the exact bound estimate (5.4). Furthermore, we have the upper estimate

$$
\operatorname{lip} S(\bar{x}, \bar{y}) \leq \operatorname{reg} Q(\bar{y}, \bar{z}) \cdot\left\|\nabla_{x} f(\bar{x}, \bar{y})\right\|
$$

provided in addition that $\widehat{\operatorname{lip}}_{y} f(\bar{x}, \bar{y})=0$.

Proof. Since $f$ is strictly partially differentiable at $(\bar{x}, \bar{y})$, condition (3.1) in Theorem 5.1 is satisfied. Furthermore, we conclude from Proposition 2.5 that conditions (5.1) and (5.2) reduce, respectively, to (5.9) and (5.10) of this corollary. The latter obviously gives (5.11) if $\widehat{\operatorname{lip}}_{y} f(\bar{x}, \bar{y})=0$. Assertion (ii) of the corollary is a direct consequence of Theorem 5.1(ii).

Similarly to Remark 3.6(ii) we observe from (5.11) and (5.12) that the exact bound equality

$$
\operatorname{lip} S(\bar{x}, \bar{y})=\operatorname{reg} Q(\bar{y}, \bar{z}) \cdot\left\|\nabla_{x} f(\bar{x}, \bar{y})\right\|
$$


holds provided that $C\left(\nabla_{x} f(\bar{x}, \bar{y})\right)=1$ for the relative condition number defined in (3.22).

Our next result establishes a characterization of the Lipschitz-like property for the solution map (1.2) via metric regularity of the sum involving the field $Q$ in the generalized equation (1.1) and a strict estimator of the base mapping $f$ with respect to $y$, which exists when, e.g., $f$ is strictly partially differentiable with respect to the decision variable. A metric space version of assertion (ii) in the following theorem is given in [5, Theorem 5E.3].

Theorem 5.3. (Lipschitz-like property of solution maps via strict base estimators.) Let $f: X \times Y \rightarrow Z$ be a mapping between Banach spaces that is Lipschitz continuous in a neighborhood $U \times V$ of $(\bar{x}, \bar{y}) \in X \times Y$, let $Q: Y \rightrightarrows Z$ be a set-valued mapping with $-f(\bar{x}, \bar{y}) \in Q(\bar{y})$, and let $h: Y \rightarrow Z$ be a strict estimator of $f$ around $(\bar{x}, \bar{y})$ with respect to $y$ uniformly in $x$ with constant $\lambda$ such that the graph $\operatorname{gph}(h+Q)$ is locally closed around $(\bar{y}, 0)$. The following assertions hold:

(i) Assume that the solution map $S$ in (1.2) is Lipschitz-like around $(\bar{x}, \bar{y})$ and that there are a surjective mapping $A \in \mathcal{L}(X, Z)$ and a constant $\mu \geq 0$ such that

$$
\operatorname{reg} A \cdot[\mu+\lambda \operatorname{lip} S(\bar{x}, \bar{y})]<1
$$

and that condition (3.1) is satisfied. Then the mapping $h+Q$ is metrically regular around $(\bar{y}, 0)$ with the exact bound estimate

$$
\operatorname{reg}(h+Q)(\bar{y}, 0) \leq \frac{\operatorname{reg} A \cdot \operatorname{lip} S(\bar{x}, \bar{y})}{1-\operatorname{reg} A \cdot[\mu+\lambda \operatorname{lip} S(\bar{x}, \bar{y})]} .
$$

(ii) Conversely, assume that $h+Q$ is metrically regular around $(\bar{y}, 0)$ and that

$$
\lambda \operatorname{reg}(h+Q)(\bar{y}, 0)<1 .
$$

Then the solution map $S$ is Lipschitz-like around $(\bar{x}, \bar{y})$ with the exact bound estimate

$$
\operatorname{lip} S(\bar{x}, \bar{y}) \leq \frac{\operatorname{reg}(h+Q)(\bar{y}, 0) \cdot \widehat{\operatorname{lip}}_{x} f(\bar{x}, \bar{y})}{1-\lambda \operatorname{reg}(h+Q)(\bar{y}, 0)} .
$$

Proof. To justify (i), we proceed similarly to the proof of assertion (i) in Theorem 5.1 applying Lemma 3.1 but with $f(x, y)$ changed now by $g(x, y):=f(x, y)-h(y)$. It is easy to see that $\widehat{\operatorname{lip}}_{y} g(\bar{x}, \bar{y})=\lambda$. Then for every $\gamma>\operatorname{reg} A$ there is a constant $\alpha>0$ such that for all points $(y, z),\left(y^{\prime}, z^{\prime}\right) \in \mathbb{B}_{\alpha}(\bar{y}) \times \mathbb{B}_{\alpha}(0)$ we have the inclusion

$$
\Upsilon\left(y^{\prime}, z^{\prime}\right) \cap \mathbb{B}_{\alpha}(\bar{x}) \subset \Upsilon(y, z)+\frac{\gamma}{1-\gamma \mu}\left(\lambda\left\|y-y^{\prime}\right\|+\left\|z-z^{\prime}\right\|\right) \mathbb{B},
$$

where $\Upsilon(y, z):=\{x \in X \mid f(x, y)-h(y)+z=0\}$. The rest of the proof follows the lines in Theorem 5.1(i) with constructing Cauchy sequences satisfying the relationships

$$
\begin{gathered}
y_{k} \in S\left(x_{k}\right), \quad\left\|y_{k}-y_{k-1}\right\| \leq \theta\left\|x_{k}-x_{k-1}\right\|, \\
x_{k} \in \Upsilon\left(y_{k-1}, z\right), \text { and }\left\|x_{k}-x_{k-1}\right\| \leq \frac{\gamma \lambda}{1-\gamma \mu}\left\|y_{k-1}-y_{k-2}\right\| .
\end{gathered}
$$

To justify the converse implication of assertion (ii), we proceed as in the proof of Theorem 5.1(ii) while defining now a set-valued mapping $\Phi: X \rightrightarrows Y$ by

$$
\Phi(u):=(h+Q)^{-1}\left(-f\left(x^{\prime}, u\right)+h(u)\right) .
$$

Then we check that all the assumptions of Theorem 2.1 are satisfied for this mapping and apply the latter theorem to find a fixed point of $\Phi$ and thus to complete the proof. 
It is worth observing that the upper estimates in both (5.15) and (5.17) cannot be generally improved; e.g., they become the equalities, for the mappings $f$ and $Q$ from Remark 3.4(ii).

Theorem 5.3 reduces to a much simpler form under the strict differentiability assumption imposed on the base mapping $f$ at the reference point. In finite dimensions the following result is given in [5, Theorem 3F.9] with the upper estimate (5.19) of the exact Lipschitzian bound.

Corollary 5.4. (Characterizing the Lipschitz-like property of solution maps to generalized equations with strictly differentiable bases.) Let $f: X \times Y \rightarrow Z$ be a mapping between Banach spaces that is strictly differentiable at $(\bar{x}, \bar{y}) \in X \times Y$, let $Q: Y \rightrightarrows Z$ be a setvalued field mapping with closed graph around $(\bar{y},-f(\bar{x}, \bar{y})) \in \operatorname{gph} Q$, and let $h: Y \rightarrow Z$ be given by $h(y):=f(\bar{x}, \bar{y})+\nabla_{y} f(\bar{x}, \bar{y})(y-\bar{y})$. The following assertions hold:

(i) If the partial derivative operator $\nabla_{x} f(\bar{x}, \bar{y}): X \rightarrow Z$ is surjective and if the solution map $S: X \rightrightarrows Y$ in (1.2) is Lipschitz-like around $(\bar{x}, \bar{y})$, then $h+Q$ is metrically regular around $(\bar{y}, 0)$ with the exact bound estimate

$$
\operatorname{reg}(h+Q)(\bar{y}, 0) \leq \operatorname{lip} S(\bar{x}, \bar{y}) \cdot\left\|\left(\nabla_{x} f(\bar{x}, \bar{y})^{*}\right)^{-1}\right\| .
$$

(ii) If conversely $h+Q$ is metrically regular around $(\bar{y}, 0)$, then the solution map $S$ is Lipschitzlike around $(\bar{x}, \bar{y})$ with the exact bound estimate

$$
\operatorname{lip} S(\bar{x}, \bar{y}) \leq \operatorname{reg}(h+Q)(\bar{y}, 0) \cdot\left\|\nabla_{x} f(\bar{x}, \bar{y})\right\| .
$$

Proof. It is easy to verify that the mapping $h: Y \rightarrow Z$ given in the corollary is a strict estimator of the base $f$ around $(\bar{x}, \bar{y})$ with respect to $y$ uniformly in $x$ under the assumed strict differentiability of $f$ at $(\bar{x}, \bar{y})$, and that the latter ensures the fulfillment of the imposed assumptions (3.1), (5.14), and (5.16) with reg $A=\left\|\left(\nabla_{x} f(\bar{x}, \bar{y})^{*}\right)^{-1}\right\|$ therein due to Proposition 2.5. Thus we get both assertions of the corollary from the corresponding ones in Theorem 5.3 with the upper estimates (5.18) and (5.19) implied by (5.15) and (5.17), respectively.

Observe similarly to (5.13) that the exact bound equality

$$
\operatorname{lip} S(\bar{x}, \bar{y})=\operatorname{reg}(h+Q)(\bar{y}, 0) \cdot\left\|\nabla_{x} f(\bar{x}, \bar{y})\right\|
$$

follows from (5.18) and (5.19) provided that in addition to all the assumptions of Corollary 5.4 we have $C\left(\nabla_{x} f(\bar{x}, \bar{y})\right)=1$ for the relative condition number defined in (3.22).

Remark 5.5. (Failure of calmness-subregularity relationships between solution maps and fields of generalized equations.) Let us illustrate by simple examples that at-point counterparts of the above relationships obtained in this section for robust Lipschitz-like and metric regularity properties do not generally hold.

(i) Consider mappings $f: \mathbb{R} \times \mathbb{R} \rightarrow \mathbb{R}$ and $Q: \mathbb{R} \rightarrow \mathbb{R}$ in (1.1) given by

$$
f(x, y):=x-y^{2} \text { and } Q(y):=y^{2} \quad \text { for } x, y \in \mathbb{R} \text {. }
$$

Take $\bar{x}=\bar{y}=0$ and define a strict estimator $h: \mathbb{R} \rightarrow \mathbb{R}$ of $f$ as in Corollary 5.4, i.e., $h \equiv 0$ in this case. Then $\nabla_{x} f(0,0)(x)=x$, which is obviously a surjective mapping from $\mathbb{R}$ to $\mathbb{R}$. We have furthermore that $\widehat{\operatorname{lip}}_{y} f(0,0)=0$, and thus all the assumptions of Corollary 5.4(i) are satisfied. It is easy to see that the corresponding solution map

$$
S(x)= \begin{cases}\mathbb{R} & \text { if } x=0 \\ \emptyset & \text { otherwise }\end{cases}
$$


is calm at $(0,0)$ while the mapping $Q \equiv h+Q$ is not metrically subregular at $(0,0)$.

(ii) The at-point counterpart of the converse implication obtained in Corollary 5.4(ii) does not hold as well. Indeed, take $Q \equiv 0$ and $f$ as in (i). Thus $Q \equiv h+Q$ is metrically subregular at $(0,0)$ while the solution map $S(x)=\{ \pm \sqrt{x}\}$ is not calm at $(0,0)$.

It turns out nevertheless that an at-point counterparts of both assertions in Theorem 5.1 hold true for a special class of separated generalized equations with solution maps given by

$$
S(x)=\{y \in Y \mid 0 \in f(x)+Q(y)\}, \quad x \in X,
$$

i.e., when base mappings do not depend on decision variables. Observe that we do not now impose the closed-graph assumption on fields $Q$ as in Theorem 5.1. Note also that we skip formulating consequences of the next theorem for generalized equations with strictly differentiable bases; this can be easily done similarly to the previous developments.

Theorem 5.6. (Equivalence between calmness of solution maps and metric subregularity of fields in separated generalized equations.) Let $f: X \rightarrow Z$ be a mapping between Banach spaces that is Lipschitz continuous on a neighborhood $U$ of $\bar{x} \in X$, and let $Q: Y \rightrightarrows Z$ be a set-valued mapping on a Banach space $Y$ with $\bar{z}:=-f(\bar{x}) \in Q(\bar{y})$ for some $\bar{y} \in Y$. The following assertions hold:

(i) Assume that there exist a surjective linear operator $A \in \mathcal{L}(X, Z)$ and a constant $\mu \geq 0$ such that $\mu \cdot \operatorname{reg} A<1$ and that

$$
\left\|f(x)-f\left(x^{\prime}\right)-A\left(x-x^{\prime}\right)\right\| \leq \mu\left\|x-x^{\prime}\right\| \text { for all } x, x^{\prime} \in U .
$$

If the solution map $S$ in (5.20) is calm at $(\bar{x}, \bar{y})$, then the field mapping $Q$ is metrically subregular at $(\bar{y}, \bar{z})$ with the exact bound estimate

$$
\text { subreg } Q(\bar{y}, \bar{z}) \leq \frac{\operatorname{clm} S(\bar{x}, \bar{y}) \cdot \operatorname{reg} A}{1-\mu \cdot \operatorname{reg} A} .
$$

(ii) Conversely, the metric subregularity of $Q$ at $(\bar{y}, \bar{z})$ implies the calmness of the solution map $S$ at $(\vec{x}, \vec{y})$ with with exact bound estimate

$$
\operatorname{lm} S(\bar{x}, \bar{y}) \leq \operatorname{lip} f(\bar{x}) \cdot \operatorname{subreg} Q(\bar{y}, \bar{z})
$$

Proof. To justify assertion (i), suppose that the solution map $S$ in $(5.20)$ is calm at $(\bar{x}, \bar{y})$ with $\operatorname{clm} S(\bar{x}, \bar{y})<\kappa$ and $\gamma>\operatorname{reg} A$ with $\gamma \mu<1$ under the assumptions made. It follows from Lemma 3.1 that there is a constant $\alpha>0$ such that we have the inclusion

$$
\Gamma\left(z^{\prime}\right) \cap \mathbb{B}_{\alpha}(\bar{x}) \subset \Gamma(z)+\frac{\gamma}{1-\gamma \mu}\left\|z-z^{\prime}\right\| \mathbb{B} \text { for every } z, z^{\prime} \in \mathbb{B}_{\alpha}(\bar{z})
$$

where $\Gamma(z):=\{x \in X \mid f(x)+z=0\}$. Make $\alpha$ smaller if necessary so that

$$
S(x) \cap \mathbb{B}_{\alpha}(\bar{y}) \subset S(\bar{x})+\kappa\|x-\bar{x}\| \mathbb{B} \text { for all } x \in \mathbb{B}_{\alpha}(\bar{x})
$$

and then take $z \in \mathbb{B}_{\alpha}(\bar{z})$ and $y \in Q^{-1}(z) \cap \mathbb{B}_{\alpha}(\bar{y})$ observing that we are done if such a point $y$ does not exist. Since $\bar{x} \in \Gamma(\bar{z})$, there is some $x \in \Gamma(z)$ with $\|x-\bar{x}\| \leq \gamma /(1-\gamma \mu)\|z-\bar{z}\|$. This gives $y \in S(x) \cap \mathbb{B}_{\alpha}(\bar{y})$, and from (5.24) we get $\widehat{y} \in S(\bar{x})$ satisfying $\|\widehat{y}-\bar{y}\| \leq \kappa\|x-\bar{x}\|$. The latter implies that $\bar{z}=-f(\bar{x}) \in Q(\widehat{y})$ with the estimate

$$
\|\widehat{y}-\bar{y}\| \leq \frac{\kappa \gamma}{1-\gamma \mu}\|x-\bar{x}\|
$$


which justifies the subregularity of $Q$ and the exact bound inequality (5.21).

To prove the converse assertion (ii), suppose now that the field mapping $Q$ is metrically subregular at $(\bar{y}, \bar{z})$ and take some $\kappa>\operatorname{subreg} Q(\bar{y}, \bar{z})$. Then there is constant $a>0$ such that $f$ is Lipschitz continuous on $\mathbb{B}_{a}(\bar{x})$ with constant $\eta>\operatorname{lip} f(\bar{x})$ and also

$$
d\left(y, Q^{-1}(\bar{z})\right) \leq \kappa d(\bar{z}, Q(y)) \text { for all } y \in \mathbb{B}_{a}(\bar{y}) .
$$

Pick further $x \in \mathbb{B}_{a}(\bar{x}) \backslash\{\bar{x}\}$ and $y \in S(x) \cap \mathbb{B}_{a}(\bar{y})$ observing that there is nothing to prove if such a point $y$ does not exist. Then $-f(x) \in Q(y)$ and thus

$$
d\left(y, Q^{-1}(\bar{z})\right) \leq \kappa d(\bar{z}, Q(y)) \leq \kappa\|f(x)-f(\bar{x})\| \leq \kappa \eta\|x-\bar{x}\| .
$$

The latter ensures that for every $\varepsilon>0$ there is $y_{\varepsilon} \in Q^{-1}(\bar{z})$ satisfying $\left\|y_{\varepsilon}-y\right\| \leq(\kappa \eta+\varepsilon)\|x-\bar{x}\|$. Hence we have $-f(\bar{x})=\bar{z} \in Q\left(y_{\varepsilon}\right)$ and therefore $y_{\varepsilon} \in S(\bar{x})$. Since $\varepsilon$ can be taken arbitrarily small, the proof of (ii) and of the whole theorem is complete.

In conclusion of the paper we establish new verifiable characterizations of calmness and metric subregularity for solution maps to generalized equations with subdifferential fields generated by convex potentials $\varphi: Y \rightarrow \overline{\mathbb{R}}$. The results obtained are expressed via the so-called quadratic growth conditions imposed on the convex potential $\varphi$ that have been used in [1] and [24] for characterizing, respectively, metric regularity and subregularity of subdifferentials and upper Lipschitzian behavior of their inverses. More specifically, our results below are based on the quadratic growth characterization of metric subregularity of subdifferentials from [1] and the equivalence results for parametric generalized equations established in this paper.

Lemma 5.7. (Characterizing metric subregularity and calmness of subdifferentials.) Let $\varphi: Y \rightarrow \overline{\mathbb{R}}$ be a lower semicontinuous convex function on a Hilbert space $Y=Y^{*}$, let $\varphi^{*}: Y \rightarrow \overline{\mathbb{R}}$ be its (Fenchel) conjugate defined by

$$
\varphi^{*}(v):=\sup _{y \in Y}\{\langle v, y\rangle-\varphi(y)\}, \quad v \in Y,
$$

and let $\bar{v} \in \partial \varphi(\bar{y})$. Then the following assertions hold:

(i) The subdifferential mapping $\partial \varphi: Y \Rightarrow Y$ is metrically subregular at $(\bar{y}, \bar{v})$ if and only if there is a neighborhood $U$ of $\bar{y}$ and a constant $\alpha>0$ such that

$$
\varphi(y) \geq \varphi(\bar{y})-\langle\bar{v}, \bar{y}-y\rangle+\alpha d^{2}\left(y,(\partial \varphi)^{-1}(\bar{v})\right) \text { for all } y \in U .
$$

(ii) The subdifferential mapping $\partial \varphi$ is calm at $(\bar{y}, \bar{v})$ if and only if there is a neighborhood $V$ of $\bar{v}$ and $a$ constant $\alpha>0$ such that

$$
\varphi^{*}(v) \geq \varphi^{*}(\bar{v})-\langle\bar{y}, \bar{v}-v\rangle+\alpha d^{2}(v, \partial \varphi(\bar{y})) \text { for all } v \in V .
$$

Proof. Assertion (i) is proved in [1, Theorem 3.3]. To justify (ii), recall the classical relationship $(\partial \varphi)^{-1}=\partial \varphi^{*}$ between the inverse subdifferential of $\varphi$ and the subdifferential of its conjugate. Thus the quadratic growth characterization (5.26) follows from the one in (5.25) by taking into account that the calmness of $\partial \varphi$ is equivalent to the metric subregularity of $(\partial \varphi)^{-1}$ at the corresponding points due to the equivalence result of Proposition 2.4(ii).

Our final statement provides quadratic growth characterizations of the metric subregularity and calmness properties of solution maps to parametric generalized equations. 
Corollary 5.8. (Quadratic growth characterizations of metric regularity and calmness of solution maps to generalized equations.) Let

$$
S(x):=\{y \in Y \mid 0 \in f(x, y)+\partial \varphi(y)\}, \quad x \in X,
$$

define the solution map of the parametric generalized equation with the Hilbert space $Y$ of decision variables and the Banach space $X$ of parameters, and let $\varphi: Y \rightarrow \mathbb{R}$ be a lower semicontinuous convex function. Given $(\bar{x}, \bar{y})$ with $\bar{v}:=-f(\bar{x}, \bar{y}) \in \partial \varphi(\bar{y})$, assume that $f: X \times Y \rightarrow Y$ is Lipschitz continuous around $(\bar{x}, \bar{y})$ and partially strictly differentiable at this point with respect to $x$ uniformly in $y$ and that its partial derivative operator $\nabla_{x} f(\bar{x}, \bar{y}): X \rightarrow Y$ is surjective. The following hold:

(i) The solution map $S$ in (5.27) is metrically subregular at $(\bar{x}, \bar{y})$ if and only if the conjugate growth condition (5.26) is satisfied.

(ii) Suppose that the base mapping $f=f(x)$ in (5.27) does not depend on the decision variable $y$. Then the solution map $S$ is calm at $(\bar{x}, \bar{y})$ if the quadratic growth condition (5.25) is satisfied.

Proof. Assertion (i) of the corollary follows from assertions (ii) of Lemma 5.7 and the equivalence between calmness of fields and metric subregularity of solution maps in generalized equations obtained in Corollary 3.5(ii). Assertion (ii) of this corollary is a consequence of Lemma 5.7(i) and the specification of Theorem 5.6 for the case of separated generalized equations with strictly differentiable base mappings.

Acknowledgements. The authors are grateful to Marco Lopez, Steve Robinson, and Terry Rockafellar for valuable discussions on the topics and results presented in this paper.

\section{References}

[1] F.J. Aragón Artacho, M.H. Geoffroy, Characterization of metric regularity of subdifferentials, J. Convex Anal. 15 (2008) 365-380.

[2] J.-P. Aubin, Lipschitz behavior of solutions to convex minimization problems, Math. Oper. Res. 9 (1984) 87-111.

[3] D. Aussel, Y. Garcia, N. Hadjisavvas, Single-directional property of multivalued maps and lack of metric regularity for variational systems, preprint, September 2008.

[4] A.V. Dmitruk, A.A. Milyutin, N.P. Osmolovskii, Lyusternik's theorem and the theory of extrema, Russian Math. Surv. 35 (1980) 11-51.

[5] A.L. Dontchev, R.T. Rockafellar, Implicit Functions and Solution Mappings, Springer, Berlin, 2009.

[6] W. Geremew, N.M. Nam, B.S. Mordukhovich, Coderivative calculus and metric regularity for constraint and variational systems, Nonlinear Anal. 70 (2009) 529-552.

[7] L.M. Graves, Some mapping theorems, Duke Math. J. 17 (1950) 111-114.

[8] A.D. Ioffe, Regular points of Lipschitz functions, Trans. Amer. Math. Soc. 251 (1979) 61-69.

[9] A.D. Ioffe, Metric regularity and subdifferential calculus, Russian Math. Surv. 55 (2000) 501558. 
[10] A.D. Ioffe, J.V. Outrata, On metric and calmness qualification conditions in subdifferential calculus, Set-Valued Anal. 16 (2008) 199-227.

[11] P. Kenderov, Semi-continuity of set-valued monotone mappings, Fund. Math. 88 (1975) 61-69.

[12] D. Klatte, B. Kummer, Nonsmooth Equations in Optimization: Regularity, Calculus, Methods and Applications, Kluwer, Boston, 2002.

[13] A.B. Levy, R.A. Poliquin, Characterizing the single-valuedness of multifunctions, Set-Valued Anal. 5 (1997) 351-364.

[14] L.A. Lyusternik, On conditional extrema of functionals, Math. Sbornik 41 (1934) 390-401.

[15] B.S. Mordukhovich, Complete characterizations of openness, metric regularity, and Lipschitzian properties of multifunctions, Trans. Amer. Math. Soc. 340 (1993) 1-35.

[16] B.S. Mordukhovich, Lipschitzian stability of constraint systems and generalized equations, Nonlinear Anal. 22 (1994) 173-206.

[17] B.S. Mordukhovich, Variational Analysis and Generalized Differentiation, I: Basic Theory, II: Applications, Springer, Berlin, 2006.

[18] B.S. Mordukhovich, Failure of metric regularity for major classes of variational systems, Nonlinear Anal. 69 (2008) 918-924.

[19] J. Renegar, Linear programming, complexity theory and elementary functional analysis, Math. Program. 70 (1995) 279-351.

[20] S.M. Robinson, Generalized equations and their solutions, I: Basic theory, Math. Program. Study 10 (1979) 128-141.

[21] S.M. Robinson, Some continuity properties of polyhedral multifunctions, Math. Program. Study 14 (1981) 206-214.

[22] S.M. Robinson, Equations on locally thin sets, Math. Oper. Res., to appear.

[23] R.T. Rockafellar, R.J-B. Wets, Variational Analysis, Springer, Berlin, 1998.

[24] R. Zhang, J. Treiman, Upper-Lipschitz multifunctions and inverse subdifferentials, Nonlinear Anal. 24 (1995) 273-286.

[25] X.Y. Zheng, K.F. Ng, Metric subregularity and constraint qualifications for convex generalized equations in Banach spaces, SIAM J. Optim. 18 (2007) 437-460. 